\title{
The Transit Light Source Effect. II. The Impact of Stellar Heterogeneity on Transmission Spectra of Planets Orbiting Broadly Sun-like Stars
}

\author{
Benjamin V. Rackham ${ }^{1,6,7}$ (1), Dániel Apai ${ }^{1,2,3,6}$ (i), and Mark S. Giampapa ${ }^{4,5}$ (10) \\ ${ }^{1}$ Department of Astronomy/Steward Observatory, The University of Arizona, 933 N. Cherry Avenue, Tucson, AZ 85721, USA; brackham@as.arizona.edu \\ ${ }^{2}$ Department of Planetary Sciences, The University of Arizona, 1629 E. University Boulevard, Tucson, AZ 85721, USA \\ ${ }^{3}$ Max Planck Institute for Astronomy, Königstuhl 17, D-69117 Heidelberg, Germany \\ ${ }^{4}$ National Solar Observatory, 950 N. Cherry Avenue, Tucson, AZ 85719, USA \\ ${ }^{5}$ Lunar and Planetary Laboratory, University of Arizona, Tucson, AZ 85711, USA \\ Received 2018 September 7; revised 2018 December 12; accepted 2018 December 14; published 2019 February 5
}

\begin{abstract}
Transmission spectra probe exoplanetary atmospheres, but they can also be strongly affected by heterogeneities in host star photospheres through the transit light source effect. Here we build upon our recent study of the effects of unocculted spots and faculae on M-dwarf transmission spectra, extending the analysis to FGK dwarfs. Using a suite of rotating model photospheres, we explore spot and facula covering fractions for varying activity levels and the associated stellar contamination spectra. Relative to $\mathrm{M}$ dwarfs, we find that the typical variabilities of FGK dwarfs imply lower spot covering fractions, though they generally increase with later spectral types, from $\sim 0.1 \%$ for $\mathrm{F}$ dwarfs to $2 \%-4 \%$ for late-K dwarfs. While the stellar contamination spectra are considerably weaker than those for typical M dwarfs, we find that typically active $\mathrm{G}$ and $\mathrm{K}$ dwarfs produce visual slopes that are detectable in high-precision transmission spectra. We examine line offsets at $\mathrm{H} \alpha$ and the $\mathrm{Na}$ and $\mathrm{K}$ doublets and find that unocculted faculae in $\mathrm{K}$ dwarfs can appreciably alter transit depths around the $\mathrm{Na} \mathrm{D}$ doublet. We find that bandaveraged transit depth offsets at molecular bands for $\mathrm{CH}_{4}, \mathrm{CO}, \mathrm{CO}_{2}, \mathrm{H}_{2} \mathrm{O}, \mathrm{N}_{2} \mathrm{O}, \mathrm{O}_{2}$, and $\mathrm{O}_{3}$ are not detectable for typically active FGK dwarfs, though stellar $\mathrm{TiO} / \mathrm{VO}$ features are potentially detectable for typically active late-K dwarfs. Generally, this analysis shows that inactive FGK dwarfs do not produce detectable stellar contamination features in transmission spectra, though active FGK host stars can produce such features, and care is warranted in interpreting transmission spectra from these systems.
\end{abstract}

Key words: methods: numerical - planets and satellites: atmospheres - planets and satellites: fundamental parameters - stars: activity - starspots - techniques: spectroscopic

\section{Introduction}

Transiting exoplanets provide an opportunity to study the atmospheres of distant worlds. During a transit, the host star illuminates the exoplanet's atmosphere, enabling measurements of the properties of the optically thin upper atmosphere. Changes in transit depth as a function of wavelength, i.e., the transmission spectrum, encode information about absorption and scattering in the exoplanet's atmosphere (Seager \& Sasselov 2000; Brown 2001; Hubbard et al. 2001). This technique has led to discoveries of atomic and molecular absorption in exoplanetary atmospheres (e.g., Charbonneau et al. 2002; Sing et al. 2012), provided constraints on their bulk metallicities (Fraine et al. 2014; Kreidberg et al. 2014a, 2015; Wakeford et al. 2017, 2018; Nikolov et al. 2018), and has recently begun to enable comparative studies of exoplanetary atmospheres (Sing et al. 2016; Barstow et al. 2017; Pinhas et al. 2018, 2019).

At the same time, photospheric heterogeneities on the host star produce wavelength-dependent effects on the transmission spectrum through the transit light source (TLS) effect. Essentially, transit observations are differential measurements that necessarily compare transit depth changes to an out-oftransit baseline. However, the out-of-transit baseline is set by the integrated stellar disk, while the actual light source for the transmission measurement is provided by the emergent

\footnotetext{
${ }^{6}$ Earths in Other Solar Systems Team, NASA Nexus for Exoplanet System Science.

${ }_{7}^{7}$ National Science Foundation Graduate Research Fellow.
}

spectrum of the spatially resolved transit chord. As a result, any spectral difference between the integrated stellar disk and the transit chord will be imprinted on the differential measurement (Pont et al. 2008; Berta et al. 2011; Sing et al. 2011; McCullough et al. 2014). Given this fundamental difference from a classical laboratory transmission measurement, in which the spectrum of the light source is well characterized, exoplanet transmission spectroscopy studies should assume that some level of TLS contamination (or "stellar" contamination) exists with every measurement and seek to place limits on it. For further context on stellar contamination of transmission spectra, we refer the reader to Apai et al. (2018).

The most prominent photospheric heterogeneities are magnetic active regions. These include spots-cool, dark regions of suppressed convection (Parker 1955; Babcock 1961) - and faculae-the hot, bright walls of flux tubes (Spruit 1976) and granules (Keller et al. 2004; Lites et al. 2004) revealed via projection effects. These active regions, i.e., spots and faculae, are ubiquitous features of stars with convective outer layers (see reviews by Ruzmaikin 2001; Berdyugina 2005; Strassmeier 2009; Collier Cameron 2017). When present within the transit chord, active regions produce time-resolved bumps in transit light curves that affect transit depth determinations (e.g., Pont et al. 2008). More perniciously, when present outside the transit chord, active regions affect transit depths through the TLS effect.

The ability of stars to imprint spectral features in transmission spectra has been recognized for more than a decade, 
mostly in the form of in-depth studies of individual exoplanet host stars (e.g., Pont et al. 2008, 2013; Bean et al. 2010; Berta et al. 2011; Sing et al. 2011; Jordán et al. 2013; Oshagh et al. 2014; Cauley et al. 2017; Rackham et al. 2017). In a systematic study of stellar contamination in $\mathrm{M}$ dwarfs (Rackham et al. 2018, hereafter Paper I), we found that rotational variability amplitudes that are typically observed for M dwarfs correspond to a wide range of spot and facula covering fractions. Accordingly, a wide uncertainty exists for the scale of the stellar contamination spectra associated with these active regions. This finding has important implications for highprecision observations of low-mass planets around $\mathrm{M}$ dwarfs, for which active regions can imprint molecular features in transmission spectra on a scale that is comparable to or even an order of magnitude larger than that of atmospheric features of small, rocky exoplanets.

In contrast to their M-dwarf counterparts, FGK dwarfs generally display lower relative amplitudes of rotational brightness variations. For example, McQuillan et al. (2014) derive rotation periods for over 34,000 main-sequence Kepler stars with effective temperatures below $6500 \mathrm{~K}$, roughly a quarter of the full Kepler sample. They find that periodic fractions decrease with increasing temperature, from 0.83 for stars in their coolest temperature bin $\left(T_{\text {eff }}<4000 \mathrm{~K}\right)$ to 0.20 for stars in their hottest bin $\left(T_{\text {eff }} \in[6000,6500] \mathrm{K}\right)$, in broad agreement with results from Basri et al. (2013). They also find that variability amplitudes decrease with increasing temperature as well, with median amplitudes of $0.7 \%$ and $0.2 \%$ for these same bins (see their Table 1 and Figure 3). These lower periodic fractions and variability amplitudes for hotter stars point to differences in the properties of magnetic active regions and suggest that FGK dwarfs generally pose fewer difficulties for transmission spectroscopy observations than cooler stars.

Yet, despite their overall lower rotational variabilities, FGK stars still present their own challenges for transmission spectroscopy. Recently, Cauley et al. (2018) examined the effects of spots and faculae on chromospherically sensitive atomic lines in high-resolution visual transmission spectra of $\mathrm{G}$ and $\mathrm{K}$ dwarfs. They explored models for four effective temperatures from 4500 to $6000 \mathrm{~K}$, corresponding to mid-K to early-G dwarfs, and ranges of spot covering fractions from $0.3 \%$ to $10 \%$ and facular covering fractions from $5 \%$ to $50 \%$. They found that large facular covering fractions can appreciably alter transit depths for $\mathrm{H} \alpha$, Ca II K, and $\mathrm{Na} \mathrm{I} \mathrm{D,} \mathrm{which}$ underscores the need to constrain active region covering fractions for active $\mathrm{G}$ and $\mathrm{K}$ dwarf hosts in order to properly interpret atomic line detections in high-resolution transmission spectra.

Observational efforts also attest to the challenges posed by FGK stars to transmission spectroscopy studies. The hot Jupiter HD 189733b, for example, demonstrates a strong blueward slope in its visual transmission spectrum, which has been interpreted as Rayleigh scattering by condensate grains in the planetary atmosphere (Lecavelier Des Etangs et al. 2008; Pont et al. 2008, 2013; Sing et al. 2011, 2016). The data used to arrive at this interpretation have been corrected for the effect of the $1 \%-2 \%$ coverage of unocculted spots that one would infer from variability monitoring of the $\mathrm{K} 1 \mathrm{~V}$ host star (Pont et al. 2013). However, if the spot coverage is instead $\sim 4 \%$, McCullough et al. (2014) showed that the observed transmission spectrum is also consistent with a clear planetary atmosphere and a larger contribution from unocculted spots.
Furthermore, some uncertainty exists as to whether the intransit $\mathrm{H} \alpha$ absorption signature has a stellar or planetary origin or some combination of both, though the lack of a clear relationship between the stellar activity level and the $\mathrm{H} \alpha$ absorption signal argues against a purely stellar origin (Cauley et al. 2017). Similarly, in a recent study of the visual transmission spectrum of the hot Jupiter WASP-19b, Espinoza et al. (2019) applied an atmospheric retrieval approach that considers both stellar and planetary spectral features and found that the TiO features observed in one of their six transits likely originate with unocculted spots on the active G9V host star, in contrast to previous planetary interpretations for the features (Sedaghati et al. 2017). These tensions in interpretations illustrate the need for a systematic study of the spectral features produced in transmission spectra by broadly Sun-like stars.

In this work, we extend our analysis of the TLS effect to investigate stellar contamination in 0.05-5.5 $\mu \mathrm{m}$ transmission spectra of exoplanets with FGK host stars. We find that stellar contamination is generally less problematic for FGK dwarfs, though potentially observable signals are possible for more active host stars, later spectral types, and observations at shorter wavelengths. Section 2 details the rotational variability model for FGK dwarfs that we use to determine spot and facula covering fractions corresponding to typical activity levels. We present in Section 3 the contamination spectra for typically active FGK dwarfs. In Section 4 we discuss the scale of the stellar contamination and examine trends in spectral features, and we summarize the key findings of this analysis in Section 5.

\section{Stellar Variability Modeling}

We modeled rotational variability amplitudes that are due to photospheric heterogeneities for $\mathrm{F}, \mathrm{G}$, and $\mathrm{K}$ dwarfs following the method detailed in Paper I. Following convention, we organized our analysis around spectral types, which is effectively the same as organizing it by effective temperature with irregular grid spacing. In the following section, we briefly summarize the methodology and detail differences in the current analysis with respect to Paper I.

\subsection{Adopted Stellar Parameters}

We generated model photospheres for spectral types F5V$\mathrm{K} 9 \mathrm{~V}$, including three photospheric components-immaculate photosphere, spots, and faculae-and parameterized them by their temperatures. We adopted the effective temperature $T_{\text {eff }}$ for each spectral type from those tabulated by Pecaut \& Mamajek (2013) and set the photosphere temperature $T_{\text {phot }}$ to this value. We linearly interpolated within the grid of solarmetallicity stellar models of Siess et al. (2000) to determine masses and radii for early main-sequence stars with these effective temperatures, with which we calculated surface gravities.

Typical starspot temperature contrasts vary as a function of stellar effective temperature, with larger temperature contrasts observed for hotter stars (Berdyugina 2005, and references therein). We fitted a linear relation to the photosphere and spot temperatures of dwarfs presented in Table 5 of Berdyugina (2005), excluding the outliers of the solar penumbra and EK Dra, and adopted the following relation for the spot temperature $T_{\text {spot }}$ as a function of $T_{\text {phot }}$ :

$$
T_{\text {spot }}=0.418 \times T_{\text {phot }}+1620 \mathrm{~K} \text {, }
$$


Table 1

Adopted Stellar Parameters

\begin{tabular}{|c|c|c|c|c|}
\hline Sp. Type & $T_{\text {phot }}(\mathrm{K})$ & $T_{\text {spot }}(\mathrm{K})$ & $T_{\text {fac }}(\mathrm{K})$ & $\log g(\mathrm{cgs})$ \\
\hline F5V & 6510 & 4340 & 6610 & 4.32 \\
\hline F6V & 6340 & 4270 & 6440 & 4.35 \\
\hline F7V & 6240 & 4230 & 6340 & 4.36 \\
\hline F8V & 6150 & 4190 & 6250 & 4.38 \\
\hline F9V & 6040 & 4140 & 6140 & 4.40 \\
\hline G0V & 5920 & 4090 & 6020 & 4.42 \\
\hline G1V & 5880 & 4080 & 5980 & 4.43 \\
\hline $\mathrm{G} 2 \mathrm{~V}$ & 5770 & 4030 & 5870 & 4.46 \\
\hline G3V & 5720 & 4010 & 5820 & 4.47 \\
\hline G4V & 5680 & 3990 & 5780 & 4.47 \\
\hline G5V & 5660 & 3980 & 5760 & 4.48 \\
\hline G6V & 5590 & 3960 & 5690 & 4.49 \\
\hline G7V & 5530 & 3930 & 5630 & 4.50 \\
\hline G8V & 5490 & 3910 & 5590 & 4.51 \\
\hline G9V & 5340 & 3850 & 5440 & 4.54 \\
\hline K0V & 5280 & 3830 & 5380 & 4.55 \\
\hline K1V & 5170 & 3780 & 5270 & 4.56 \\
\hline $\mathrm{K} 2 \mathrm{~V}$ & 5040 & 3730 & 5140 & 4.58 \\
\hline K3V & 4840 & 3640 & 4940 & 4.61 \\
\hline $\mathrm{K} 4 \mathrm{~V}$ & 4620 & 3550 & 4720 & 4.64 \\
\hline $\mathrm{K} 5 \mathrm{~V}$ & 4450 & 3480 & 4550 & 4.67 \\
\hline K6V & 4200 & 3370 & 4300 & 4.73 \\
\hline K7V & 4050 & 3310 & 4150 & 4.78 \\
\hline K8V & 3970 & 3280 & 4070 & 4.81 \\
\hline K9V & 3880 & 3240 & 3980 & 4.85 \\
\hline
\end{tabular}

in which both temperatures are given in Kelvin.

Following Gondoin (2008), we adopted faculae temperatures of $T_{\mathrm{fac}}=T_{\mathrm{phot}}+100 \mathrm{~K}$. For comparison, Kobel et al. (2011) find an average contrast of $3.7 \%$ in quiet-Sun network magnetic elements with a broad range of about $-15 \%$ to $+10 \%$. A $3.7 \%$ contrast on the Sun would correspond to an increase of roughly $50 \mathrm{~K}$ over the photosphere, so we find the simple scaling relation that we adopt to be suitable. We note, however, that this simple relation neglects the complex dependence of facular contrast on magnetic field strength and limb distance (Norris et al. 2017), which we save for consideration in a future work.

Table 1 lists the adopted surface gravities and photosphere, spot, and faculae temperatures for each spectral type. We note that the relation that we adopt for $T_{\text {spot }}$ is determined using a stellar sample with effective temperatures between $3300 \mathrm{~K}$ (M3) and $5870 \mathrm{~K}$ (G1) (Berdyugina 2005, and references therein) and so may not hold for F dwarfs. However, in their study of rotation periods for main-sequence Kepler targets with $T_{\text {eff }}<6500 \mathrm{~K}$, McQuillan et al. (2014) detect rotational variability for 4318 dwarfs with $T_{\text {eff }} \in[5980,6500] \mathrm{K}$ (see their Table 1), corresponding to spectral types F5V-F9V. They find a periodic detection fraction of 0.20 for stars with $T_{\text {eff }} \in[6000,6500] \mathrm{K}$, similar to the fractions for stars with $T_{\text {eff }} \in[5000,5500] \mathrm{K}(0.27)$ and $T_{\text {eff }} \in[5500,6000] \mathrm{K}(0.16)$. We interpret this as evidence that the physical mechanism that drives rotational variability in $\mathrm{G}$ and $\mathrm{K}$ dwarfs, i.e., starspots and faculae, extends to stars as hot as F5 dwarfs. Therefore, we adopt the scaling relation in Equation (1) for our full sample of spectral types.

For additional context, we briefly review in Section 2.1.1 the literature on $\mathrm{F}$ dwarf photospheric features.

\subsubsection{Note on F Dwarf Parameters}

Going from hotter to cooler stars, chromospheric and coronal emission first occurs on the main sequence in the F dwarf stars, for which models of stellar structure also predict the onset of outer convection zones. In particular, a sharp increase in the detection rate of stellar X-ray emission is seen at $(B-$ $V) \sim 0.3$, coinciding with late-A to early- $\mathrm{F}$ main-sequence stars (Schmitt 2001). While magnetic activity is clearly present, little is known about the morphology of the emergent magnetic flux regions in the photospheres of $\mathrm{F}$ dwarfs. However, longterm monitoring programs in $\mathrm{Ca}$ II $\mathrm{H}$ and $\mathrm{K}$ and Strömgren photometry suggest a rather homogeneous spatial distribution of magnetic regions on $\mathrm{F}$ dwarfs.

Noyes et al. (1984) included 34 F stars (see their Table 1) in their study of rotation, convection, and activity on the main sequence, based on early results from intensive monitoring of the $\mathrm{H}$ and $\mathrm{K}$ lines to detect rotational modulation. Of these, only 7 objects exhibited rotational modulation in their $\mathrm{H}$ and $\mathrm{K}$ lines, while no periodic variability was seen in the remaining 27 stars, even though chromospheric activity in this sample was enhanced by an average factor of 1.5 compared to the Sun. In their summary of the cycle properties of the stars in the Mt. Wilson Survey, Baliunas et al. (1995) included $40 \mathrm{~F}$ stars in their sample (see their Table 2). Of these, definitive cycle periods were measured in only 10 objects, which were all $\sim$ F5 or later. Broadband photometric observations are consistent with the results from the $\mathrm{H}$ and $\mathrm{K}$ monitoring in the context of apparent departures from axisymmetric distributions of magnetic regions. In particular, Radick et al. (1982) found that photometric variability was not present in stars earlier than $\sim$ F7 at a detection limit of $0.5 \%$. Thus, $\mathrm{F}$ stars are characterized by a distinct lack of departures from axial symmetry of their surface distributions of magnetic flux.

The direct measurement of magnetic field properties on these stars has proven challenging because of their relatively more rapid rotation ${ }^{8}$ and the apparent absence of large-scale fields that typically give rise to spectrophotometric modulations. As discussed by Giampapa \& Rosner (1984), the relatively higher angular velocities of $\mathrm{F}$ dwarfs results in the generation of flux ropes at the base of the thin convection zone that are characterized by small spatial scales (Schmitt \& Rosner 1983). Following magnetic flux rope dynamo generation, only minimal expansion of the emergent flux ropes is expected to occur. Thus, even though magnetic activity may be enhanced, large-scale inhomogeneities do not necessarily occur. The transition between this behavior in the limit of thin convection zones to Sun-like, "thick" convection zones must occur at about F7V because it is near this spectral type that photometric variability begins to appear, at least as documented in groundbased observations.

Finally, we note that high activity in the form of X-ray or $\mathrm{Ca}$ II emission can be present in $\mathrm{F}$ dwarfs even if only lowamplitude photometric variability is present. For example, Pizzolato et al. (2003, Table 4) show that saturated X-ray emission with $\log L_{X} \sim 30.1-30.3$ occurs for $F$ stars later than F5. Schröder et al. (2009, Figure 13) find enhanced $\log R_{\mathrm{HK}}^{\prime}$ values relative to the Sun in F dwarfs, but with a declining envelope of values toward early-F dwarfs, where convection

\footnotetext{
8 This rapid rotation of $\mathrm{F}$ dwarfs can introduce equator-to-pole temperature gradients (Deupree 2011), which represent a distinct source of stellar contamination that we do not consider in detail here.
} 

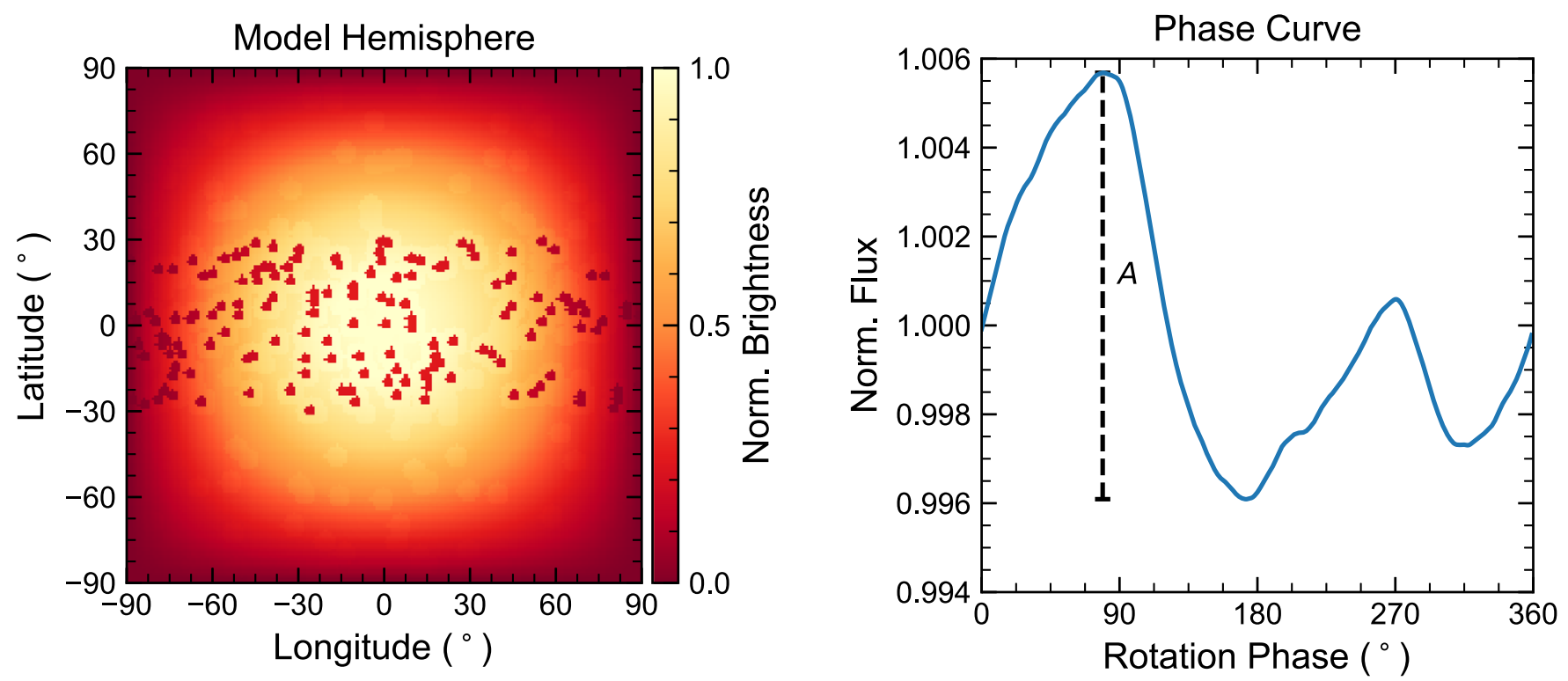

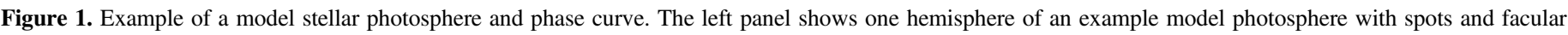

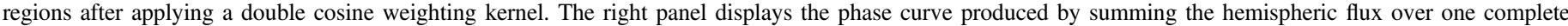

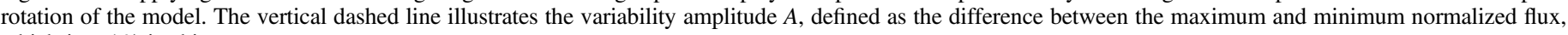
which is $\sim 1 \%$ in this case.

zones are thinning out. Therefore, on one hand, low photometric variability among $\mathrm{F}$ dwarfs does not necessarily mean low magnetic activity. On the other hand, however, it is clear that activity is decreasing toward spectral types earlier than about F5-F7.

\subsection{Stellar Spectral Components}

For each spectral type, we generated spectra for the immaculate photosphere, spots, and faculae using the PHOENIX stellar spectral model grid (Husser et al. 2013). We used models with solar metallicity $([\mathrm{Fe} / \mathrm{H}]=0.0)$ and no $\alpha$-element enrichment $([\alpha / \mathrm{Fe}]=0.0)$. The PHOENIX grid provides highresolution spectra covering $0.05-5.5 \mu \mathrm{m}$ for effective temperatures in steps of $100 \mathrm{~K}$ for $T_{\text {eff }} \in[2300,7000] \mathrm{K}$ and $200 \mathrm{~K}$ for $T_{\text {eff }} \in[7000,12000] \mathrm{K}$. Surface gravities are provided in steps of 0.5 for $\log g \in[0.0,6.0]$. We linearly interpolated within the model grid in terms of temperature and surface gravity to produce component spectra with the parameters detailed in Table 1.

\subsection{Rotational Variability Model}

We employed the rotational modeling approach detailed in Paper I to investigate the range of photospheric heterogeneities consistent with observed variabilities. The approach involves iteratively adding active regions to a model photosphere and recording the peak-to-trough variability amplitude $A$ that results from rotating the model after each addition, as illustrated in Figure 1. Active regions are added at random coordinates until the model photosphere has the desired maximum spot coverage. The entire process is repeated 100 times to build up statistics on the dependence of the variability amplitude on the spot covering fraction.

We refer the reader to Paper I for a detailed description of the variability model and provide here the specifics for this work. However, one important assumption of this model bears repeating. As in Paper I, we assume that the stellar rotation axis is aligned with the plane of the sky. This assumption is good for most transiting exoplanet systems because the presence of transits indicates a nearly edge-on planetary orbit, and obliquities between the stellar rotation axis and planetary orbital plane are generally $\lesssim 20^{\circ}$ (Winn et al. 2017). The principal exceptions to this rule are hot stars with hot Jupiters, which tend to have high obliquities (Schlaufman 2010; Winn et al. 2010a; Albrecht et al. 2012; Mazeh et al. 2015). The threshold stellar temperature above which these systems have a broader obliquity distribution is $6090_{-110}^{+150} \mathrm{~K}$ (Dawson 2014), which roughly coincides with the "Kraft break" that separates cool stars with convective envelopes from hot stars with radiative envelopes (Kraft 1967) and the boundary between F and $\mathrm{G}$ spectral types for our adopted parameters (Table 1). This suggests that determining spot and facula covering fractions from variability amplitudes for individual F-dwarf systems with hot Jupiters may require a more detailed treatment of the obliquity than the simple assumption that we make here. Nonetheless, as we find the TLS spectral signals produced by $\mathrm{F}$ dwarfs to be relatively minor compared to those for later spectral types (see Section 4), we make this assumption for all models in this study and note that more detailed models may be required to investigate active region coverages in individual F-dwarf systems of interest or other notably oblique systems, such as the HAT-P-11 system (Winn et al. 2010b; Hirano et al. 2011; Yee et al. 2018).

As in Paper I, we used a model photosphere with a resolution of $180 \times 360$ pixels. We simulated the immaculate photosphere, spots, and faculae by setting the pixel values to the flux of the component spectra integrated over the Kepler instrument response function. This allows us to directly compare the rotational variabilities from our models to those reported by McQuillan et al. (2014). We fixed the spot size to $R_{\text {spot }}=2^{\circ}$ so that each spot covered $400 \mathrm{ppm}$ of a projected hemisphere (13 resolution elements), which is similar to large spot groups on the Sun (Mandal et al. 2017). While a detailed history of facular observations exists for the Sun (e.g., Makarov \&

\footnotetext{
http://keplergo.arc.nasa.gov/Instrumentation.shtml
} 
Makarova 1996; Shapiro et al. 2014), little is known about the prevalence, distribution, and temperature contrasts for faculae on other stars. ${ }^{10}$ Given this considerable uncertainty, we considered cases both with and without faculae. We refer to these hereafter as the spots+faculae and spots cases, respectively. For the spots+faculae models, we added faculae at the 10:1 facula-to-spot area ratio observed for the active Sun (Shapiro et al. 2014), half of which were associated with spots and half of which were located independently, following the approach detailed in Paper I.

Spots on the Sun are found at active latitudes that vary predictably over the course of a solar cycle (Maunder 1904), giving rise to the well-known butterfly diagram (Maunder 1922). While individual sunspots can appear at latitudes as high as $\pm 40^{\circ}-50^{\circ}$, sunspot locations generally start around $28^{\circ}$ from the equator at the beginning of a solar cycle and drift toward the equator over the course of a cycle (Hathaway 2011). Spots on the K4 dwarf HAT-P-11 have a mean latitude of $\approx 16^{\circ} \pm 1^{\circ}$ and are generally found within $30^{\circ}$ of the equator (Morris et al. 2017), which illustrates that the active latitudes observed on the Sun apply to at least some mid-K dwarfs as well. Faculae, on the other hand, are not confined to equatorial regions on the Sun; they can be found associated in spots or alone in polar regions (Makarov \& Makarova 1996). Following these results, we restricted the locations of spots but not faculae to latitudes within $30^{\circ}$ of the equator.

For each spectral type, we generated 100 model photospheres and added spots (and faculae) to each at randomly selected coordinates until we reached a full-disk spot covering fraction of $33 \%$. This is the maximum spot coverage possible for our models, given the restriction on the spot latitudes. From the set of 100 models, we calculated the mean and standard deviation of the variability amplitude as a function of spot covering fraction.

Finally, we note that adopting a Sun-like spot distribution in our models may cause us to underestimate the spot coverages and thus TLS signals from stars with notably different spot distributions. Polar spots, for example, are commonly observed in Doppler images of rapidly rotating stars (see Strassmeier 2009, and references therein). If present on an exoplanet host star, such a spot configuration would contribute little to the rotational variability while producing a relatively large TLS signal. While our intent here is to investigate typical active region coverages and TLS signals for Sun-like stars, studies of exoplanet host stars with suspected nonsolar active region distributions could benefit from other approaches such as stellar spectral decomposition (e.g., Neff et al. 1995; Gully-Santiago et al. 2017) and simultaneous retrievals of stellar and planetary properties in transmission spectra (e.g., Pinhas et al. 2018; Espinoza et al. 2019). We discuss some of these techniques in Section 4.9.

\subsection{Variability as a Function of Spot Covering Fraction}

We find that the variability amplitudes for each spectral type behave as a predictable function of the spot covering fraction. Variability amplitudes grow with increasing spot coverages until they reach a maximum near $16.5 \%$ - at which point half of the photosphere within the allowed latitudes is covered in

\footnotetext{
${ }^{10}$ Observations of transiting exoplanets offer a promising probe of stellar photospheres that can shed light on this problem, however (e.g., Dravins et al. 2017a, 2017b, 2018; Rackham et al. 2017; Espinoza et al. 2019).
}

spots-and then decline as the spot coverages continue to increase to the maximum spot covering fraction and the equatorial band completely fills with spots. The behavior is roughly symmetric about spot coverages of $16.5 \%$ and similar for both spots and spots+faculae models.

As a result, variability amplitudes near the maximum amplitude correspond to a range of spot covering fractions, while smaller variability amplitudes correspond to two distinct spot covering fractions. Therefore, the relatively low variability amplitudes considered in this work (see Section 2.5) have both low and high spot coverage solutions. In this study, we are primarily interested in studying the extent of photospheric heterogeneities and the associated stellar contamination spectra for typical FGK stars. While spot coverages of $33 \%$ and higher have been identified for young and/or active stars such as LkCa 4 (Gully-Santiago et al. 2017), we consider the turnover in variability amplitudes above $16.5 \%$ spot coverage and the relatively low variabilities associated with nearly $33 \%$ spot coverage to be artifacts of our model prescriptions. Accordingly, we restrict our analysis to spot covering fractions below $16.5 \%$. We note that a more realistic model would allow for a wider latitudinal spot distribution for very active stars (i.e., those with spot covering fractions $10 \%$ and higher).

Figure 2 shows the variability amplitudes as a function of spot covering fraction for all spectral types that we consider. The relationship between variability amplitudes and spot covering fractions appears similar for spots and spots +faculae models. In both cases the variability amplitudes grow with a square-root-like dependence on the spot covering fraction. However, the variability amplitudes grow more slowly for low spot covering fractions in the spots+faculae models than in the spots models, indicating that the presence of faculae tends to suppress the rotational variability.

These results contrast with those on $\mathbf{M}$ dwarfs, which show that the addition of faculae leads to large initial increases in variability amplitudes and larger amplitudes overall for the maximum spot covering fractions (Paper I). This difference results from our model assumptions: we adopt a fixed temperature difference between the facula and immaculate photosphere components, which causes the facular contrast to decrease with increasing photosphere temperatures. The contrast is largest for $\mathrm{M}$ dwarfs and smallest for $\mathrm{F}$ dwarfs. This shows that including faculae in the models leads to large initial increases in variability for the coolest stars. Additionally, for spot covering fractions above $\sim 10 \%$, the spots+faculae models are completely covered by either spots or faculae, given the 10:1 facula-to-spot area ratio that we adopt. For M dwarfs, the contrast between spots and faculae is notably larger than that between spots and immaculate photosphere, which causes the overall larger variability amplitudes for the spots +faculae models. For FGK stars, on the other hand, the spots/photosphere and spots/faculae contrasts are more comparable, resulting in the similar amplitudes for the spots and spots+faculae models.

The apparent square-root-like dependence of the variability amplitude on the spot covering fraction can be understood as a consequence of the random longitudinal distribution of the spots. For the case in which only spots contribute to the photospheric heterogeneity, the maximum brightness during a rotation will be at the longitude with the fewest spots, and the minimum brightness will be at the longitude with the most spots. As the spots are distributed randomly in longitude, the 


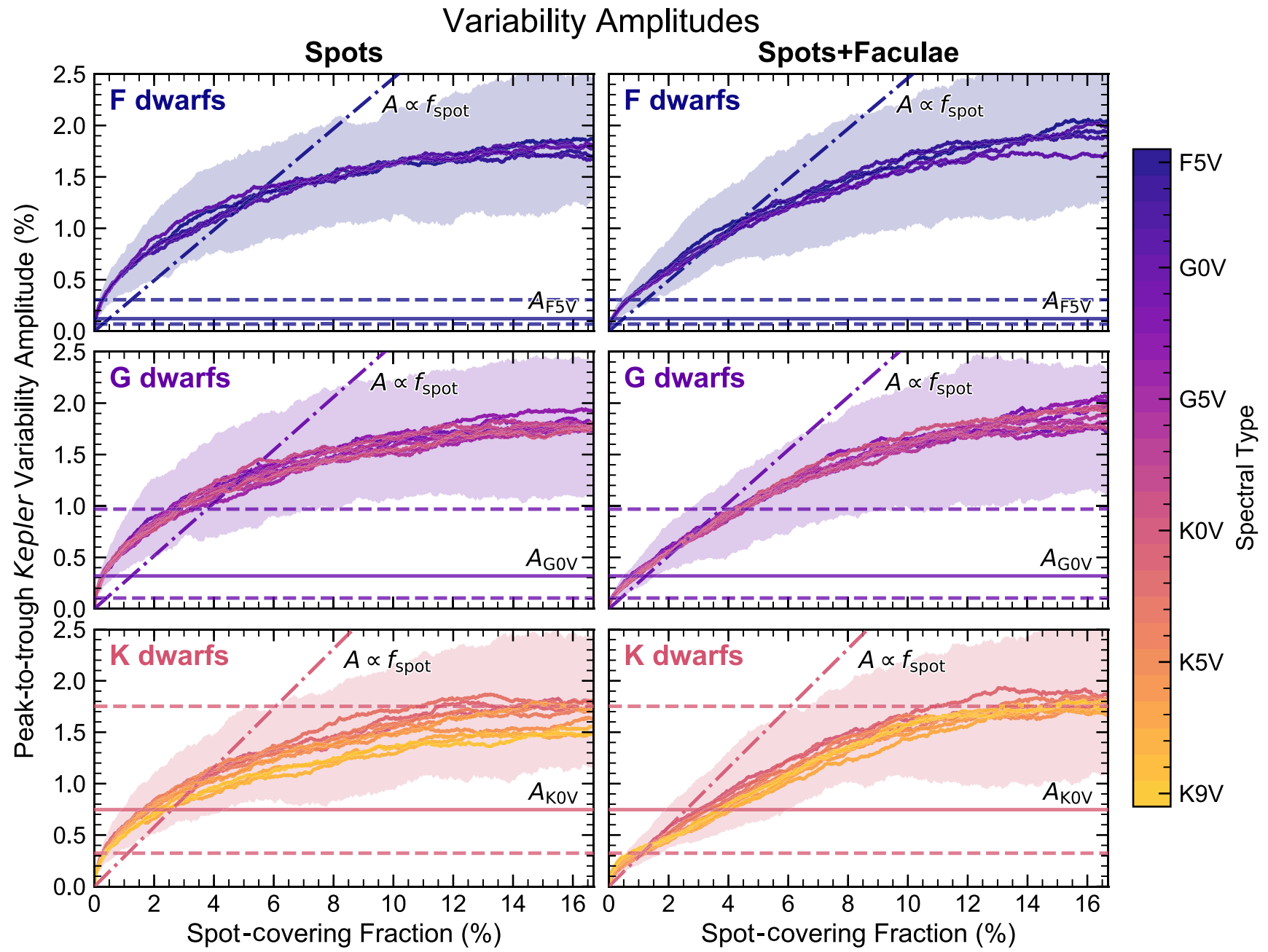

Figure 2. Variability amplitudes as a function of spot covering fraction for spots (left) and spots ffaculae (right) models. The top, middle, and bottom rows illustrate the results of models for F, G, and K main-sequence spectral types, respectively. Solid curves give the mean variability as a function of spot covering fraction, color-coded by spectral type. For the earliest spectral type in each panel, the shaded region indicates the range encompassing $68 \%$ of the model outcomes, which is comparable to the dispersion in model outcomes for all other spectral types. The dash-dotted line shows the expected scaling of spot covering fraction and variability for this spectral type, given its Kepler-band photosphere and spot fluxes and assuming a linear relation. For all models, the linear relation is clearly not the appropriate scaling between the spot covering fraction and photometric variability, although it is a less poor approximation than in the case of M dwarfs (Paper I). Additionally, the horizontal lines show the median (solid line) and the 16th and 84th percentiles (dashed lines) of variability amplitudes for Kepler dwarf stars for this spectral type (McQuillan et al. 2014). In each case, the variability grows asymptotically as a function of $f_{\text {spot }}$ until it reaches a maximum near $16.5 \%$, at which point half of the equatorial band is populated with spots and the photosphere is maximally heterogeneous. The dispersion in model outcomes leads to a range of spot covering fractions that correspond to a given amplitude.

expectation value for this difference for given number of spots $n$ will be on the order of $\sqrt{n}$, and the expectation value for the rotational variability amplitude will be on the order of $\alpha \Omega \sqrt{n}$, where $\alpha$ is the spot contrast $\left(1-F_{\text {spot }} / F_{\text {phot }}\right)^{11}$ and $\Omega$ is the solid angle of the spot. The dependence of $A$ on $f_{\text {spot }}$ should therefore scale roughly as $\sqrt{f_{\text {spot }}}$, since $n \sim f_{\text {spot. }}$. For a given value of $f_{\text {spot }}$, the relation should be steeper for larger spot contrasts and sizes. Of course, the presence of faculae complicates this picture, as they can occur in association with spots or in isolated regions, and their brightness contribution thus weakens the relationship between spot coverage and longitudinal brightness. The exact analytical dependence of $A$ on $f_{\text {spot }}$ (and $f_{\text {fac }}$ ) will depend on these parameters as well as on inclination and limb-darkening effects (as pointed out by Jackson \& Jeffries 2012); a full derivation of it is beyond the scope of this analysis but could yield interesting insights in a

\footnotetext{
${ }^{11}$ In the Kepler bandpass, the values of $\alpha$ for the models we use vary (nonmonotonically) from 0.86 for F0V to 0.73 for K9V.
}

future study. For the present, we refer the reader to Jackson \& Jeffries (2013) for a more detailed discussion of the relation between variability amplitude and spot filling factor, typical size, and contrast.

Given the apparent square-root-like dependence of the variability amplitudes on the spot covering fraction, we fit via least squares a scaling relation of the form

$$
A=C \times f_{\text {spot }}^{0.5}
$$

to the variability amplitudes of the spots and spots +faculae models for each spectral type, following Paper I. In this expression, $C$ is a scaling coefficient that depends on the properties of the active regions and determines the amplitude of the relation. Table 2 provides the fitted values of $C$ with uncertainties that reflect the 68\% dispersion in variability amplitudes illustrated by the shaded regions in Figure 2. These 
Table 2

Scaling Relation Coefficients for Variability Models

\begin{tabular}{|c|c|c|}
\hline \multirow{2}{*}{ Sp. Type } & \multicolumn{2}{|c|}{$C$} \\
\hline & spots & spots+faculae \\
\hline$\overline{\mathrm{F} 5 \mathrm{~V}}$ & $0.050 \pm 0.018$ & $0.051 \pm 0.020$ \\
\hline F6V & $0.049 \pm 0.020$ & $0.050 \pm 0.020$ \\
\hline F7V & $0.049 \pm 0.019$ & $0.051 \pm 0.019$ \\
\hline F8V & $0.050 \pm 0.019$ & $0.049 \pm 0.020$ \\
\hline F9V & $0.050 \pm 0.020$ & $0.047 \pm 0.018$ \\
\hline G0V & $0.050 \pm 0.019$ & $0.047 \pm 0.018$ \\
\hline G1V & $0.049 \pm 0.019$ & $0.050 \pm 0.019$ \\
\hline G2V & $0.050 \pm 0.019$ & $0.050 \pm 0.019$ \\
\hline G3V & $0.053 \pm 0.022$ & $0.050 \pm 0.019$ \\
\hline G4V & $0.047 \pm 0.018$ & $0.047 \pm 0.018$ \\
\hline G5V & $0.049 \pm 0.019$ & $0.048 \pm 0.018$ \\
\hline G6V & $0.048 \pm 0.018$ & $0.048 \pm 0.018$ \\
\hline G7V & $0.050 \pm 0.018$ & $0.049 \pm 0.019$ \\
\hline G8V & $0.051 \pm 0.020$ & $0.047 \pm 0.018$ \\
\hline G9V & $0.048 \pm 0.018$ & $0.051 \pm 0.019$ \\
\hline K0V & $0.049 \pm 0.018$ & $0.048 \pm 0.019$ \\
\hline $\mathrm{K} 1 \mathrm{~V}$ & $0.049 \pm 0.018$ & $0.050 \pm 0.018$ \\
\hline $\mathrm{K} 2 \mathrm{~V}$ & $0.051 \pm 0.019$ & $0.047 \pm 0.019$ \\
\hline K3V & $0.048 \pm 0.020$ & $0.047 \pm 0.018$ \\
\hline $\mathrm{K} 4 \mathrm{~V}$ & $0.048 \pm 0.019$ & $0.046 \pm 0.018$ \\
\hline $\mathrm{K} 5 \mathrm{~V}$ & $0.045 \pm 0.017$ & $0.045 \pm 0.016$ \\
\hline K6V & $0.047 \pm 0.018$ & $0.045 \pm 0.018$ \\
\hline $\mathrm{K} 7 \mathrm{~V}$ & $0.042 \pm 0.017$ & $0.043 \pm 0.017$ \\
\hline K8V & $0.041 \pm 0.015$ & $0.046 \pm 0.017$ \\
\hline K9V & $0.041 \pm 0.016$ & $0.046 \pm 0.017$ \\
\hline
\end{tabular}

can be used to estimate spot covering fractions from observed variabilities of FGK main-sequence stars.

The values of $C$ show that the variability amplitudes are similar between the spots and spots+faculae models, which illustrates that faculae do not strongly affect the rotational variability amplitudes. This finding is in agreement with results from the Sun, for which the signal from spots dominates the rotational brightness variations as viewed in the ecliptic plane (Shapiro et al. 2016). ${ }^{12}$

\subsection{Amplitude of Typical Activity Level}

In order to investigate typical levels of stellar contamination on transmission spectra, we must adopt a reference variability amplitude to use when estimating typical active region covering fractions. For the Sun, the disk passage of spots can decrease the total solar irradiance by as much as $\sim 0.3 \%$, while faculae can increase it by $0.1 \%$ (Willson et al. 1986). Turning to a wider sample, McQuillan et al. (2014) investigated periodic photometric variability amplitudes for the full Kepler sample of main-sequence stars, building upon early analyses that focused on early subsets of the Kepler data (Basri et al. 2010, 2011), specific spectral types (McQuillan et al. 2013), or exoplanet candidate host stars (Walkowicz \& Basri 2013). For the full sample including spectral types F5V-M4V, they find a median amplitude, defined as the range between the 5th and 95th percentile of normalized flux, of $\sim 5600 \mathrm{ppm}$ or $0.56 \%$,

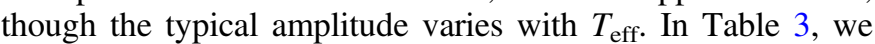
summarize the data in their Table 1 for spectral types F5VK9V separately, defining the spectral types by the outlined $T_{\text {eff }}$

\footnotetext{
${ }^{12}$ By contrast, Shapiro et al. (2016) also find that faculae dominate the longterm brightness variability on cycle timescales in the Sun and Sun-like stars for wavelengths shorter than $1.2 \mu \mathrm{m}$, regardless of viewing inclination.
}

Table 3

Median and $1 \sigma$ Range of Variability Amplitudes from McQuillan et al. (2014) by Spectral Type Bins

\begin{tabular}{|c|c|c|c|c|}
\hline \multirow{2}{*}{ Sp. Type } & \multirow{2}{*}{$\begin{array}{c}T_{\text {eff }}(\mathrm{K}) \\
\text { Range }\end{array}$} & \multirow{2}{*}{$N_{\text {bin }}$} & \multicolumn{2}{|c|}{ Variability Amplitude } \\
\hline & & & Median (\%) & $1 \sigma(\%)$ \\
\hline F5V & {$[6425,6575)$} & 373 & 0.12 & {$[0.07,0.31]$} \\
\hline F6V & {$[6290,6425)$} & 855 & 0.13 & {$[0.07,0.36$} \\
\hline F7V & {$[6195,6290)$} & 832 & 0.16 & {$[0.07,0.44]$} \\
\hline F8V & {$[6095,6195)$} & 847 & 0.21 & {$[0.09,0.61]$} \\
\hline F9V & {$[5980,6095)$} & 1411 & 0.25 & {$[0.08,0.76]$} \\
\hline G0V & {$[5900,5980)$} & 1099 & 0.32 & {$[0.10,0.97]$} \\
\hline G1V & {$[5825,5900)$} & 1106 & 0.37 & {$[0.14,1.06]$} \\
\hline $\mathrm{G} 2 \mathrm{~V}$ & {$[5745,5825)$} & 1409 & 0.41 & {$[0.14,1.18]$} \\
\hline G3V & {$[5700,5745)$} & 754 & 0.41 & {$[0.17,1.17]$} \\
\hline G4V & {$[5670,5700)$} & 633 & 0.46 & {$[0.18,1.19]$} \\
\hline G5V & {$[5625,5670)$} & 839 & 0.51 & {$[0.19,1.34]$} \\
\hline G6V & {$[5560,5625)$} & 1379 & 0.50 & {$[0.21,1.42]$} \\
\hline G7V & {$[5510,5560)$} & 1121 & 0.56 & {$[0.23,1.48]$} \\
\hline G8V & {$[5415,5510)$} & 1926 & 0.61 & {$[0.26,1.56$} \\
\hline G9V & {$[5310,5415)$} & 2267 & 0.67 & {$[0.31,1.66]$} \\
\hline $\mathrm{K} 0 \mathrm{~V}$ & {$[5225,5310)$} & 1703 & 0.75 & {$[0.32,1.75]$} \\
\hline $\mathrm{K} 1 \mathrm{~V}$ & {$[5105,5225)$} & 2162 & 0.73 & {$[0.34,1.68]$} \\
\hline $\mathrm{K} 2 \mathrm{~V}$ & {$[4940,5105)$} & 2737 & 0.76 & {$[0.36,1.71]$} \\
\hline $\mathrm{K} 3 \mathrm{~V}$ & {$[4730,4940)$} & 2560 & 0.73 & {$[0.36,1.60]$} \\
\hline $\mathrm{K} 4 \mathrm{~V}$ & {$[4535,4730)$} & 1550 & 0.69 & {$[0.37,1.49]$} \\
\hline K5V & {$[4325,4535)$} & 1415 & 0.72 & {$[0.37,1.46]$} \\
\hline K6V & {$[4125,4325)$} & 1793 & 0.67 & {$[0.35,1.28]$} \\
\hline $\mathrm{K} 7 \mathrm{~V}$ & {$[4010,4125)$} & 799 & 0.68 & {$[0.37,1.23]$} \\
\hline K8V & {$[3925,4010)$} & 449 & 0.63 & {$[0.36,1.16]$} \\
\hline K9V & {$[3865,3925)$} & 272 & 0.62 & {$[0.36,1.22]$} \\
\hline
\end{tabular}

\section{Median and $1 \sigma$ Range of Variability Amplitudes}

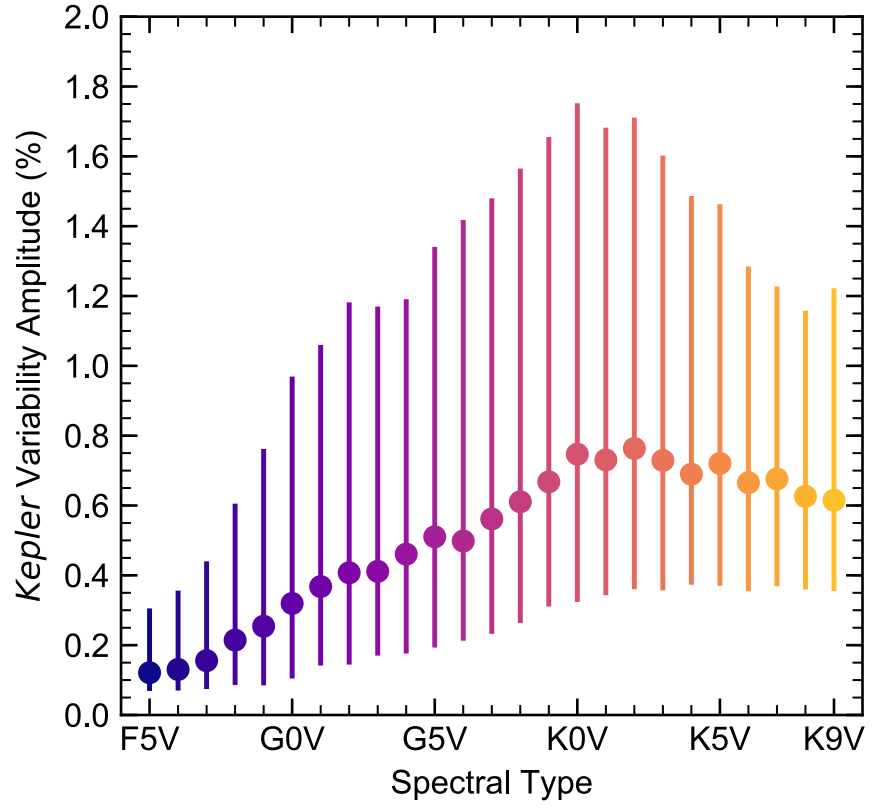

Figure 3. Medians (points) and 68\% ranges (error bars) of Kepler variability amplitudes by spectral type. Data are summarized from McQuillan et al. (2014) and color-coded by spectral type, following Figure 2. See also Table 3.

ranges. We provide the median and $1 \sigma$ (16th to 84 th percentile) range of amplitudes for each spectral type bin. These values are also illustrated in Figure 3. Later spectral types show higher variability amplitudes on average. Median variability amplitudes are highest and $1 \sigma$ ranges widest for late- $\mathrm{G}$ and early-K dwarfs. We define a "typically active" star as one showing a 
Table 4

Covering Fractions for Reference Activity Level as Determined by Variability Models

\begin{tabular}{|c|c|c|c|}
\hline \multirow{2}{*}{ Sp. Type } & \multirow{2}{*}{$\begin{array}{r}\text { spots } \\
f_{\text {spot }}(\%)\end{array}$} & \multicolumn{2}{|c|}{ spots+faculae } \\
\hline & & $f_{\text {spot }}(\%)$ & $f_{\text {fac }}(\%)$ \\
\hline F5V & $0.1_{-0.1}^{+0.1}$ & $0.1_{-0.1}^{+0.2}$ & $\overline{1_{-1}^{+2}}$ \\
\hline F6V & $0.1_{-0.1}^{+0.1}$ & $0.1_{-0.1}^{+0.2}$ & $1_{-1}^{+2}$ \\
\hline F7V & $0.1_{-0.1}^{+0.1}$ & $0.2_{-0.1}^{+0.2}$ & $2_{-1}^{+2}$ \\
\hline F8V & $0.1_{-0.1}^{+0.2}$ & $0.3_{-0.1}^{+0.5}$ & $3_{-1}^{+4}$ \\
\hline F9V & $0.2_{-0.1}^{+0.3}$ & $0.5_{-0.2}^{+0.6}$ & $5_{-2}^{+6}$ \\
\hline G0V & $0.3_{-0.1}^{+0.5}$ & $0.9_{-0.4}^{+0.7}$ & $8_{-3}^{+6}$ \\
\hline G1V & $0.4_{-0.2}^{+0.7}$ & $1.0_{-0.5}^{+1.1}$ & $10_{-4}^{+9}$ \\
\hline G2V & $0.5_{-0.2}^{+0.9}$ & $1.1_{-0.5}^{+1.1}$ & $10_{-4}^{+8}$ \\
\hline G3V & $0.4_{-0.2}^{+0.8}$ & $1.2_{-0.5}^{+1.1}$ & $11_{-4}^{+9}$ \\
\hline G4V & $0.6_{-0.3}^{+1.2}$ & $1.5_{-0.6}^{+1.5}$ & $14_{-5}^{+10}$ \\
\hline G5V & $0.7_{-0.4}^{+1.0}$ & $1.8_{-0.7}^{+2.0}$ & $16_{-5}^{+12}$ \\
\hline G6V & $0.8_{-0.4}^{+1.0}$ & $1.7_{-0.7}^{+1.5}$ & $15_{-6}^{+10}$ \\
\hline G7V & $1.0_{-0.5}^{+1.6}$ & $2.1_{-0.8}^{+2.3}$ & $18_{-6}^{+13}$ \\
\hline G8V & $1.0_{-0.4}^{+1.7}$ & $2.4_{-0.9}^{+1.9}$ & $20_{-6}^{+10}$ \\
\hline G9V & $1.3_{-0.6}^{+2.4}$ & $2.5_{-0.8}^{+2.4}$ & $21_{-5}^{+12}$ \\
\hline $\mathrm{K} 0 \mathrm{~V}$ & $1.8_{-0.9}^{+2.6}$ & $3.0_{-1.1}^{+2.9}$ & $24_{-7}^{+13}$ \\
\hline K1V & $1.7_{-0.9}^{+3.3}$ & $3.0_{-1.0}^{+2.5}$ & $24_{-7}^{+12}$ \\
\hline $\mathrm{K} 2 \mathrm{~V}$ & $1.7_{-0.8}^{+2.9}$ & $3.5_{-1.2}^{+3.6}$ & $26_{-7}^{+14}$ \\
\hline K3V & $1.8_{-0.9}^{+3.4}$ & $3.2_{-1.1}^{+2.9}$ & $25_{-7}^{+13}$ \\
\hline $\mathrm{K} 4 \mathrm{~V}$ & $1.4_{-0.7}^{+2.6}$ & $3.3_{-1.2}^{+2.9}$ & $26_{-7}^{+13}$ \\
\hline $\mathrm{K} 5 \mathrm{~V}$ & $1.7_{-0.8}^{+3.5}$ & $3.8_{-1.6}^{+3.1}$ & $28_{-9}^{+12}$ \\
\hline K6V & $1.4_{-0.7}^{+2.7}$ & $3.5_{-1.5}^{+2.3}$ & $27_{-9}^{+10}$ \\
\hline $\mathrm{K} 7 \mathrm{~V}$ & $2.1_{-1.2}^{+3.4}$ & $3.6_{-1.2}^{+3.3}$ & $27_{-7}^{+13}$ \\
\hline K8V & $1.6_{-0.8}^{+3.6}$ & $3.1_{-1.4}^{+2.6}$ & $25_{-9}^{+12}$ \\
\hline K9V & $1.5_{-0.8}^{+2.8}$ & $2.9_{-0.9}^{+2.8}$ & $23_{-6}^{+13}$ \\
\hline
\end{tabular}

rotational variability amplitude in the Kepler bandpass equal to the median for its spectral type. Accordingly, we adopt the median amplitudes from Table 3 as the reference amplitudes $A_{\text {ref }}$ that we use to determine the spot and facula covering fractions corresponding to the typical activity level for each spectral type.

\subsection{Spot and Facula Covering Fractions for Reference Amplitude}

Table 4 details and Figure 4 illustrates the active region covering fractions corresponding to the reference amplitude for each spectral type. For each set of models, the mean covering fraction consistent with $A_{\text {ref }}$ is given. The uncertainties reflect the range of covering fractions that are consistent with $A_{\text {ref }}$ for $68 \%$ of the models. In Figure 2, this range is illustrated as the intersection of $A_{\text {ref }}$ and the shaded $1 \sigma$ envelopes for the variability amplitudes from the models. Since $A$ and its $1 \sigma$ envelope grow with a square-root-like dependence on $f_{\text {spot }}$, this intersection produces uncertainties that are asymmetric and larger on the higher end.

Considering the spots models, we find that the reference variability levels are consistent with mean spot covering fractions of $0.1 \%-2.1 \%$ with $1 \sigma$ ranges that are comparable to the means. Generally, spot covering fractions are larger and their $1 \sigma$ ranges are wider for later spectral types. For the spots+faculae models, spot covering fractions are systematically higher than those from the spots models, though the values are consistent within their uncertainties. As expected, given our model assumptions, facula covering fractions are
Covering Fractions for Reference Activity Levels
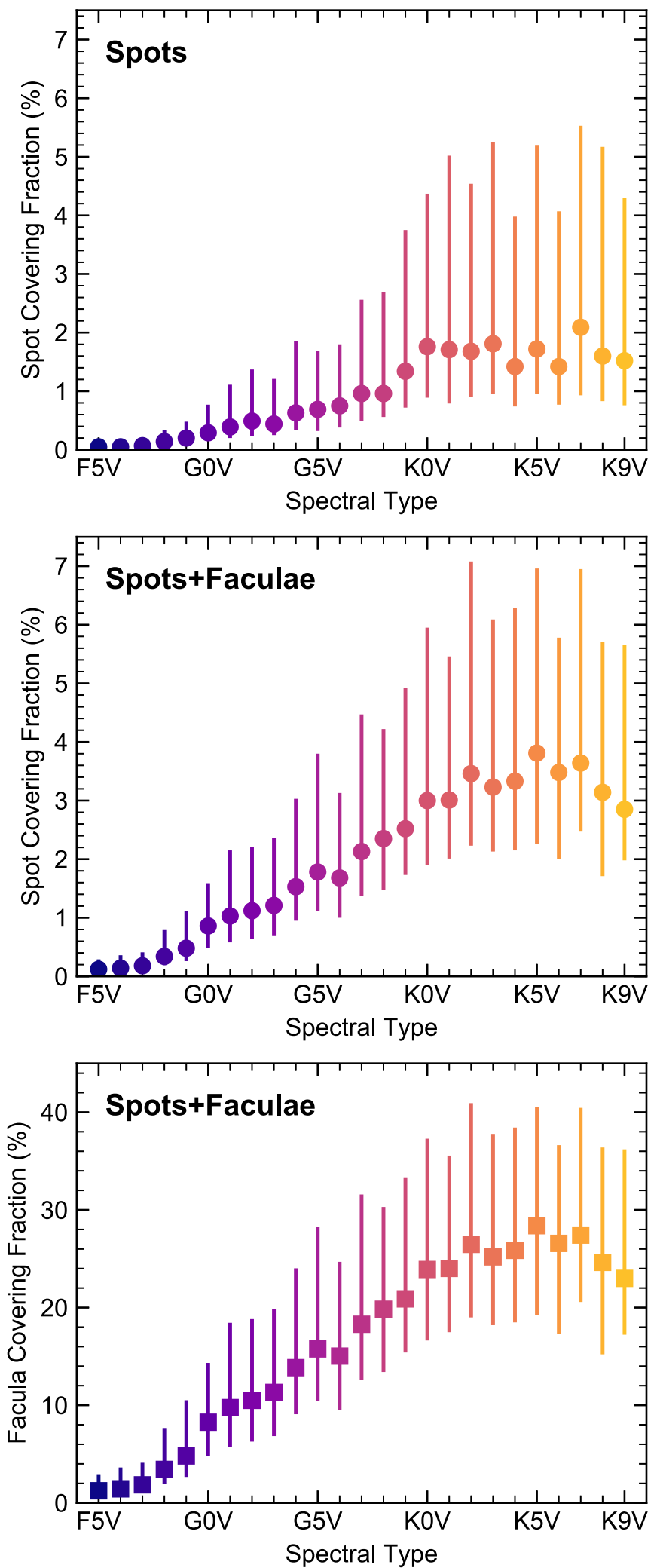

Figure 4. Active region covering fractions corresponding to reference activity levels by spectral type. Spot covering fractions for the spots models are shown in the top panel. For the spots+faculae models, spot (faculae) covering fractions are shown in the middle (bottom) panel. The data are colorcoded by spectral type, following Figure 2. See also Table 4. 
roughly 10 times larger than their spotted counterparts. In effect, faculae dampen the rotational variability produced by spots at low activity levels, allowing larger covering fractions to be consistent with the same reference variability level. This effect is visible in Figure 2, in which the relations between spot coverage and variability for the spots+faculae models are shifted to the right with respect to those of the spots models.

\section{Stellar Contamination Analysis}

With the active region covering fractions identified by the variability modeling, we can explore the typical levels of stellar contamination that we should expect for transmission spectra of exoplanets with FGK host stars.

\subsection{Model for Stellar Contamination Spectra}

We calculate the stellar contamination signal in the exoplanet transmission spectrum following the approach detailed in Paper I. In short, we take the covering fractions identified in Section 2 and, assuming the exoplanet does not transit any active regions, calculate their effect on the observed transmission spectrum using the same stellar spectral components as detailed above.

For the spots case, the stellar contamination spectrum is given by

$$
\epsilon_{\lambda, s}=\frac{1}{1-f_{\text {spot }}\left(1-\frac{S_{\lambda, \text { spot }}}{S_{\lambda, \text { phot }}}\right)},
$$

in which $S_{\lambda, \text { spot }}$ and $S_{\lambda \text {,phot }}$ are the spot and immaculate photosphere spectra, respectively (see also McCullough et al. 2014; Rackham et al. 2017; Zellem et al. 2017). For the spots +faculae case, the stellar contamination spectrum is given by

$$
\epsilon_{\lambda, s+f}=\frac{1}{1-f_{\text {spot }}\left(1-\frac{S_{\lambda, \text { spot }}}{S_{\lambda, \text { phot }}}\right)-f_{\text {fac }}\left(1-\frac{S_{\lambda, \text { fac }}}{S_{\lambda, \text { phot }}}\right)},
$$

in which $S_{\lambda, \text { fac }}$ is the facula spectra, and the remaining terms have the same meaning as above.

In general, for the case in which the planet occults a nominal emergent spectrum $S_{\lambda, 0}$ and $n$ other spectral components with covering fractions $f_{1}, f_{2}, \ldots, f_{n}$ are present elsewhere on the projected stellar disk, the stellar contamination spectrum is given by

$$
\epsilon_{\lambda, n}=\frac{1}{1-\sum_{i=1}^{n} f_{i}\left(1-\frac{S_{\lambda, i}}{S_{\lambda, 0}}\right)} .
$$

This expression is algebraically exact for a transit of a disk of uniform intensity. Since stellar disks actually display intensity profiles, it is an approximation to the true physical effect. In greater detail, the emergent spectrum of the transit chord is most important near mid-transit, and its characteristics will therefore depend on the impact parameter of the transit. Likewise, the contrast of the $n$th spectral component with the nominal spectral component $S_{\lambda, n} / S_{\lambda, 0}$ will depend on the wavelength-dependent intensity profile of the stellar disk, which can produce limb darkening or brightening or be relatively negligible at some wavelengths (e.g., Claret 2000), and the location of the $n$th component. We are interested here in examining the scale of the TLS effect for FGK stars generally, and so we ignore these higher-order effects, though in-depth studies involving precise observations of individual systems could benefit from considering them.

In all cases, $\epsilon_{\lambda}$ represents a multiplicative change to the true transit depth (i.e., the square of the wavelength-dependent planet-to-star radius ratio $\left.D_{\lambda}=\left(R_{\lambda, p} / R_{s}\right)^{2}\right)$ owing to the heterogeneity of the stellar photosphere. This combines with the planetary signal to produce the observed transit depth:

$$
D_{\lambda, \mathrm{obs}}=\epsilon_{\lambda} D_{\lambda} .
$$

As noted above, this calculation assumes that the light source illuminating the exoplanet atmosphere is described well by a single spectral component, $S_{\lambda, \text { phot }}$. Of course, spots or faculae may be present within the transit chord as well in some cases. This formalism still applies to these cases as long as the heterogeneities within the transit chord produce crossing events with amplitudes that are larger than the observation uncertainty, which allows them to be identified and taken into account (e.g., Pont et al. 2008; Carter et al. 2011; Narita et al. 2013). In fact, crossing events are useful for understanding the stellar contamination of the transmission spectrum because they enable constraints on the sizes and contrasts of active regions (Sanchis-Ojeda \& Winn 2011; Huitson et al. 2013; Mancini et al. 2013; Pont et al. 2013; Tregloan-Reed et al. 2013; Scandariato et al. 2017; Bixel et al. 2019; Espinoza et al. 2019). Still, active regions may be present within the transit chord with contrasts or sizes that do not allow them to be readily detected (Mallonn et al. 2018). More complicated models considering the distributions of heterogeneities both inside and outside the transit chord may be warranted by observations of more active host stars (e.g., Zhang et al. 2018), though this additional complication is beyond the scope of this work.

\subsection{Stellar Contamination Spectra}

Figure 5 illustrates the stellar contamination spectra that correspond to the reference variability levels for each spectral type. For the spots models, we find that unocculted spots consistent with $A_{\text {ref }}$ increase transit depths at all wavelengths studied. The contamination spectra steadily increase with decreasing wavelengths for wavelengths shorter than $\sim 1.7 \mu \mathrm{m}$, producing apparently blueward slopes. Late-K dwarf contamination spectra contain markedly more structure than their earlier spectral type counterparts. In general, the scale of the contamination spectra increases for later spectral types. The $1 \sigma$ prediction intervals on the contamination spectra, dictated by the $68 \%$ range of $f_{\text {spot }}$ and illustrated by the shaded regions in Figure 5, are asymmetric, comparable to the absolute transit depth change (i.e., $\left.\left|\epsilon_{\lambda}-1\right|\right)$ on the upper end and roughly half that value on the lower end.

The contamination spectra for the spots+faculae models are generally similar to those of the spots models but show strong differences at wavelengths shorter than $\sim 1.5 \mu \mathrm{m}$. As with the spots model, the primary effect of the stellar contamination is to increase transit depths over most of the wavelengths studied. However, owing to the presence of unocculted faculae, these spectra do not display the slopes seen at visual wavelengths with the spots models. Instead, they are relatively flat from the near-infrared (NIR) to wavelengths as short as $\sim 0.5 \mu \mathrm{m}$ and then decrease sharply. Later spectral types begin to show these decreases at longer wavelengths. For late-K dwarfs, strong decreases in transit depth are possible 


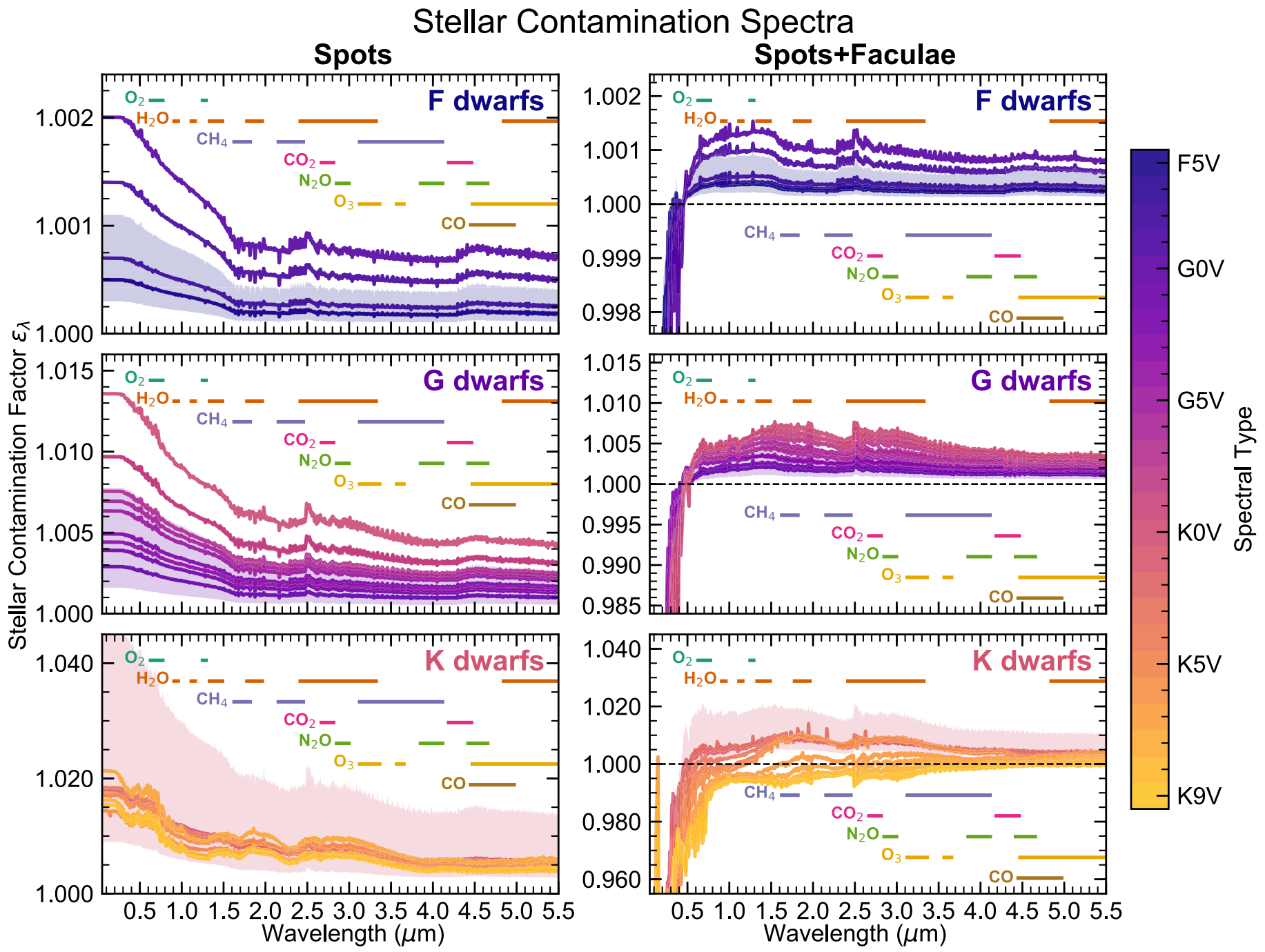

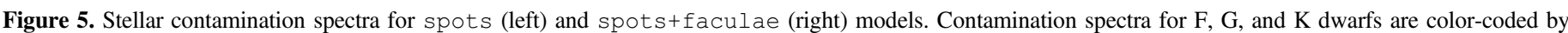

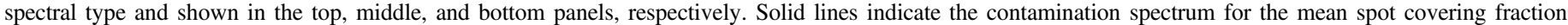

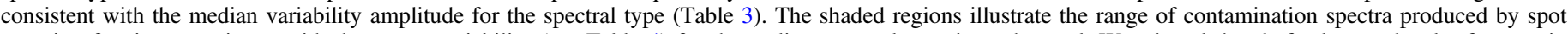

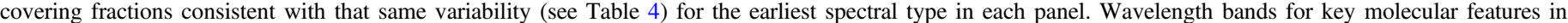
exoplanetary atmospheres are given. Note the different $y$-axis scales.

across visual wavelengths; the effect of unocculted faculae can even overwhelm that of unocculted spots to produce decreases in transit depth over the full wavelength range studied.

Thus, considering exoplanet host stars with typical activity levels, we find that $\mathrm{G}$ dwarfs produce stellar contamination signals that are a factor of a few larger than those of $\mathrm{F}$ dwarfs, while typically active $\mathrm{K}$ dwarfs produce signals that are more than an order of magnitude larger. Unocculted faculae can partially cancel out the effect of unocculted spots at visual wavelengths and can lead to large decreases in transit depths at ultraviolet (UV) wavelengths. We compare the scale of these stellar contamination signals to those of observational precisions and planetary atmospheric features in Section 4.1.

Finally, we note that for all spectral types and stellar contamination models, the most significant effects are present at the shortest wavelengths. This suggests that UV transit observations can therefore be used to place constraints on unocculted heterogeneities that affect transmission spectra more subtlety at longer wavelengths. However, the stellar models used for this analysis lack chromospheres, which contribute significantly to emergent spectra at UV wavelengths, so a considerable level of uncertainty exists for the UV contamination spectra presented here. Additionally, this picture is complicated by the temporal variability of transit depths that is due to changing stellar activity levels (e.g., Llama \& Shkolnik 2015). We discuss the impact of chromospheres further in Section 4.8 .

\subsubsection{Visual Stellar Contamination Spectra}

Visual contamination features are of particular interest in the present study, given the availability of visual transmission spectra from both ground- and space-based facilities and the increased ability of stellar active regions to contaminate visual measurements. Figure 6 provides a closer look at the features in the stellar contamination spectra at visual wavelengths. For the spots models, the contamination spectra for $F$ and $G$ dwarfs show blueward slopes and few other spectral features. Slight features are evident at wavelengths of atomic absorption for exoplanet atmospheres, namely $\mathrm{Na} \mathrm{I}, \mathrm{H} \alpha$, and $\mathrm{K}$, which we explore further in Section 4. K dwarfs, on the other hand, present more varied contamination spectra, with notable 


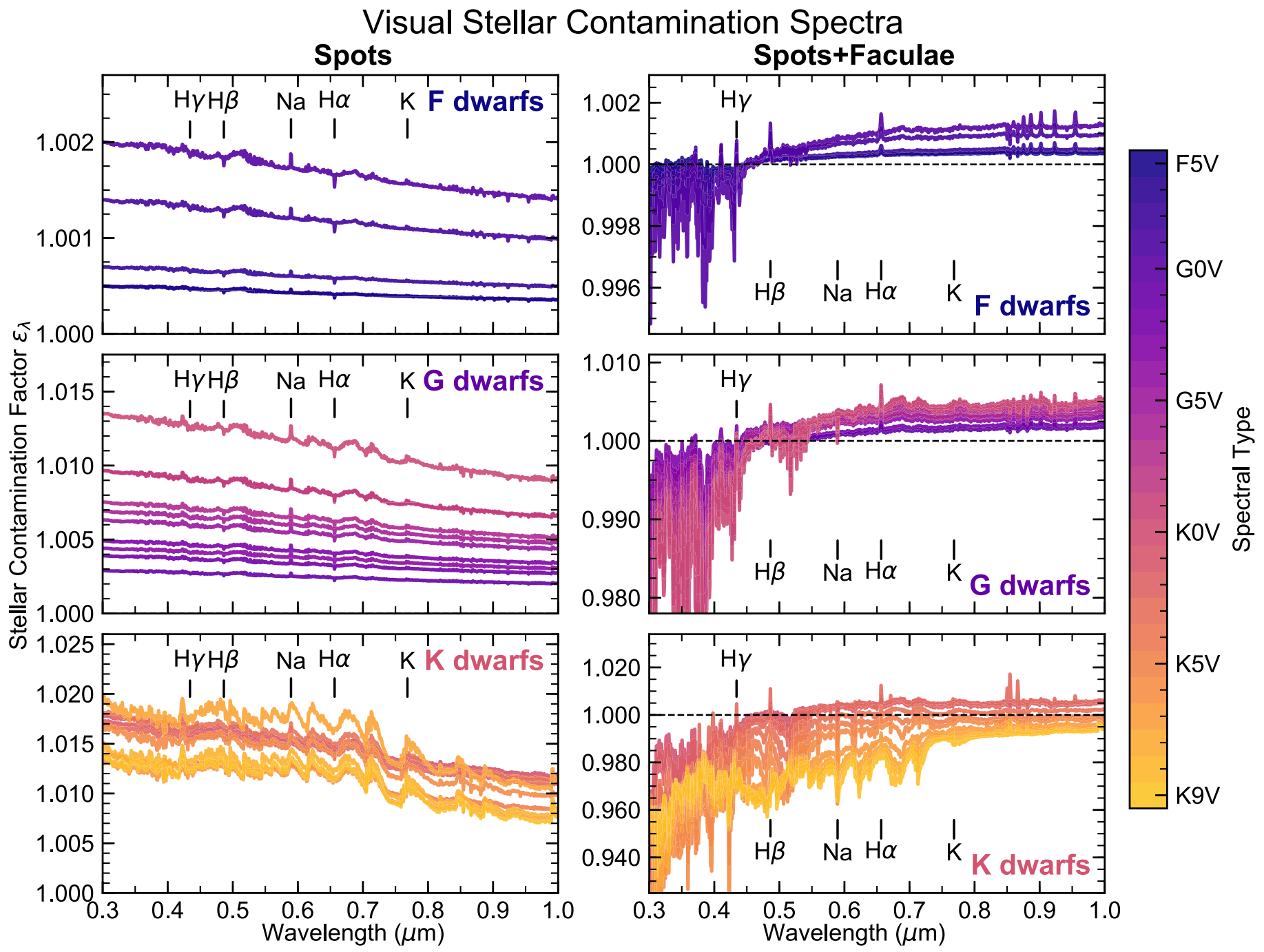

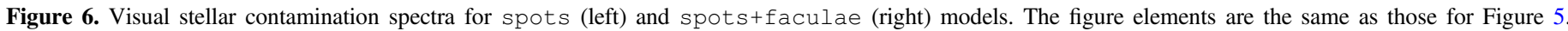
Prediction intervals on the spectra are suppressed for clarity. Key atomic absorption lines are indicated. Note the varying $y$-axis scales.

increases in transit depth around $\mathrm{TiO}$ molecular absorption features across visual wavelengths. These features are strongest for later K dwarfs.

Turning to the spotstfaculae models, we see in Figure 6 that the blueward slopes of the spots models are muted by the presence of faculae, leading to contamination spectra that are generally flat for most visible wavelengths and decrease notably for wavelengths $\lesssim 0.5 \mu \mathrm{m}$. Still, $\epsilon_{\lambda}$ remains systematically $>1$ for $\mathrm{F}$ and $\mathrm{G}$ stars at NIR wavelengths. Late$\mathrm{K}$ dwarfs are again the exception because their contamination spectra tend to decrease across all visible wavelengths and possess notably stronger spectral features than those of earlier spectral types.

\section{Discussion}

\subsection{Scale of the Stellar Contamination}

We first examine how the scale of the stellar contamination compares to that of observational precisions. We consider UV, visual, and NIR wavelengths separately due to the distinct behaviors exhibited by the contamination spectra and the different observational approaches that are used to study these wavelength regimes. Accordingly, we define the UV stellar contamination factor $\epsilon_{\mathrm{UV}}$, the visual stellar contamination factor $\epsilon_{\mathrm{vis}}^{-}$, and the NIR contamination factor $\epsilon_{\mathrm{NIR}}^{-}$as the means of the contamination spectra for wavelength ranges $0.05-0.4 \mu \mathrm{m}, 0.4-0.9 \mu \mathrm{m}$, and $0.95-5.5 \mu \mathrm{m}$, respectively. These values are provided in Table 5 for all FGK contamination spectra that we calculate, along with $1 \sigma$ prediction intervals calculated by taking the means of their $1 \sigma$ estimates (the shaded regions in Figure 5).

For the spots models, the effects of stellar contamination are more pronounced at shorter wavelengths and for later spectral types. For $\mathrm{F}$ dwarfs, we find the mean values of $\epsilon_{\mathrm{UV}}$, $\epsilon_{\mathrm{vis}}^{-}$, and $\epsilon_{\mathrm{NIR}}^{-}$are $1.0010,1.0009$, and 1.0004, respectively, all of which point to relatively minor increases in transit depths. For $\mathrm{G}$ dwarfs, the corresponding means are 1.0069, 1.0058, and 1.0028 , respectively, and for K dwarfs they are 1.0164, 1.0133, and 1.0066 .

In the NIR, the scale of the contamination spectra for the spots+faculae models is comparable to that of the spots models. The mean value of $\epsilon_{\mathrm{NIR}}$ is $1.0005,1.0032$, and 1.0030 for $\mathrm{F}, \mathrm{G}$, and $\mathrm{K}$ dwarfs, respectively. By contrast, the corresponding means at visual wavelengths are smaller (1.0004, 1.0016, and 0.9908 for F, G, and $\mathrm{K}$ dwarfs, respectively) than those of the spots models and point to 
Table 5

Mean Values of Stellar Contamination Spectra at UV, Visual, and NIR Wavelengths

\begin{tabular}{|c|c|c|c|c|c|c|}
\hline \multirow{2}{*}{ Sp. Type } & \multicolumn{3}{|c|}{ spots } & \multicolumn{3}{|c|}{ spots+faculae } \\
\hline & $\epsilon_{\overline{\mathrm{UV}}}$ & $\epsilon_{\mathrm{vis}}^{-}$ & $\epsilon_{\mathrm{NIR}}$ & $\epsilon_{\overline{\mathrm{UV}}}$ & $\epsilon_{\mathrm{vis}}^{-}$ & $\epsilon_{\overline{\mathrm{NIR}}}$ \\
\hline$\overline{\mathrm{F} 5 \mathrm{~V}}$ & $1.0005_{-0.0002}^{+0.0006}$ & $1.0004_{-0.0002}^{+0.0005}$ & $1.0002_{-0.0001}^{+0.0003}$ & $0.9960_{+0.0017}^{-0.0054}$ & $1.0002_{-0.0001}^{+0.0004}$ & $1.0003_{-0.0001}^{+0.0004}$ \\
\hline F6V & $1.0005_{-0.0001}^{+0.0005}$ & $1.0004_{-0.0001}^{+0.0004}$ & $1.0002_{-0.0001}^{+0.0002}$ & $0.9947_{+0.0022}^{-0.0077}$ & $1.0002_{-0.0001}^{+0.0004}$ & $1.0003_{-0.0001}^{+0.0005}$ \\
\hline F7V & $1.0007_{-0.0002}^{+0.0009}$ & $1.0006_{-0.0002}^{+0.0008}$ & $1.0003_{-0.0001}^{+0.0004}$ & $0.9930_{+0.0031}^{-0.0082}$ & $1.0003_{-0.0001}^{+0.0004}$ & $1.0004_{-0.0002}^{+0.0005}$ \\
\hline F8V & $1.0014_{-0.0006}^{+0.0020}$ & $1.0012_{-0.0005}^{+0.0017}$ & $1.0006_{-0.0002}^{+0.0008}$ & $0.9868_{+0.0056}^{-0.0151}$ & $1.0006_{-0.0003}^{+0.0010}$ & $1.0007_{-0.0003}^{+0.0010}$ \\
\hline F9V & $1.0020_{-0.0008}^{+0.0028}$ & $1.0017_{-0.0007}^{+0.0024}$ & $1.0008_{-0.0003}^{+0.0012}$ & $0.9797_{+0.0088}^{-0.0215}$ & $1.0007_{-0.0003}^{+0.0013}$ & $1.0010_{-0.0005}^{+0.0014}$ \\
\hline G0V & $1.0029_{-0.0013}^{+0.0048}$ & $1.0024_{-0.0011}^{+0.0041}$ & $1.0012_{-0.0005}^{+0.0020}$ & $0.9640_{+0.0143}^{-0.0228}$ & $1.0004_{-0.0003}^{+0.0011}$ & $1.0015_{-0.0007}^{+0.0015}$ \\
\hline G1V & $1.0039_{-0.0019}^{+0.0073}$ & $1.0033_{-0.0016}^{+0.0061}$ & $1.0016_{-0.0008}^{+0.0030}$ & $0.9575_{+0.0166}^{-0.0312}$ & $1.0006_{-0.0005}^{+0.0023}$ & $1.0018_{-0.0009}^{+0.0025}$ \\
\hline G2V & $1.0049_{-0.0025}^{+0.0089}$ & $1.0041_{-0.0021}^{+0.0075}$ & $1.0020_{-0.0010}^{+0.0036}$ & $0.9332_{+0.0233}^{-0.0366}$ & $1.0022_{-0.0011}^{+0.0035}$ & $1.0025_{-0.0011}^{+0.0028}$ \\
\hline G3V & $1.0044_{-0.0019}^{+0.0078}$ & $1.0037_{-0.0016}^{+0.0066}$ & $1.0018_{-0.0008}^{+0.0032}$ & $0.9301_{+0.0240}^{-0.0370}$ & $1.0016_{-0.0009}^{+0.0032}$ & $1.0024_{-0.0011}^{+0.0028}$ \\
\hline G4V & $1.0063_{-0.0029}^{+0.0124}$ & $1.0053_{-0.0025}^{+0.0105}$ & $1.0026_{-0.0012}^{+0.0051}$ & $0.9202_{+0.0234}^{-0.0398}$ & $1.0018_{-0.0011}^{+0.0045}$ & $1.0030_{-0.0013}^{+0.0038}$ \\
\hline G5V & $1.0069_{-0.0037}^{+0.0102}$ & $1.0058_{-0.0031}^{+0.0086}$ & $1.0028_{-0.0015}^{+0.0042}$ & $0.9111_{+0.0249}^{-0.0447}$ & $1.0022_{-0.0013}^{+0.0070}$ & $1.0036_{-0.0015}^{+0.0054}$ \\
\hline G6V & $1.0075_{-0.0037}^{+0.0107}$ & $1.0063_{-0.0031}^{+0.0090}$ & $1.0031_{-0.0015}^{+0.0043}$ & $0.9099_{+0.0277}^{-0.0386}$ & $1.0014_{-0.0010}^{+0.0040}$ & $1.0033_{-0.0015}^{+0.0036}$ \\
\hline G7V & $1.0096_{-0.0047}^{+0.0164}$ & $1.0081_{-0.0040}^{+0.0138}$ & $1.0039_{-0.0019}^{+0.0066}$ & $0.8877_{+0.0274}^{-0.0474}$ & $1.0019_{-0.0014}^{+0.0081}$ & $1.0042_{-0.0017}^{+0.0064}$ \\
\hline G8V & $1.0096_{-0.0040}^{+0.0178}$ & $1.0081_{-0.0034}^{+0.0149}$ & $1.0039_{-0.0017}^{+0.0072}$ & $0.8664_{+0.0311}^{-0.0378}$ & $1.0021_{-0.0017}^{+0.0064}$ & $1.0047_{-0.0020}^{+0.0051}$ \\
\hline G9V & $1.0134_{-0.0063}^{+0.0251}$ & $1.0113_{-0.0052}^{+0.0209}$ & $1.0055_{-0.0026}^{+0.0101}$ & $0.8044_{+0.0181}^{-0.0314}$ & $1.0018_{-0.0015}^{+0.0086}$ & $1.0050_{-0.0019}^{+0.0068}$ \\
\hline $\mathrm{K} 0 \mathrm{~V}$ & $1.0177_{-0.0088}^{+0.0274}$ & $1.0148_{-0.0074}^{+0.0228}$ & $1.0072_{-0.0036}^{+0.0109}$ & $0.8001_{+0.0247}^{-0.0316}$ & $1.0022_{-0.0022}^{+0.0119}$ & $1.0061_{-0.0026}^{+0.0087}$ \\
\hline K1V & $1.0172_{-0.0093}^{+0.0349}$ & $1.0143_{-0.0078}^{+0.0289}$ & $1.0070_{-0.0038}^{+0.0139}$ & $0.8080_{+0.0277}^{-0.0353}$ & $1.0004_{-0.0016}^{+0.0086}$ & $1.0058_{-0.0023}^{+0.0070}$ \\
\hline $\mathrm{K} 2 \mathrm{~V}$ & $1.0168_{-0.0079}^{+0.0299}$ & $1.0140_{-0.0065}^{+0.0247}$ & $1.0068_{-0.0032}^{+0.0119}$ & $0.8596_{+0.0305}^{-0.0415}$ & $1.0010_{-0.0024}^{+0.0150}$ & $1.0063_{-0.0028}^{+0.0107}$ \\
\hline K3V & $1.0180_{-0.0086}^{+0.0360}$ & $1.0149_{-0.0072}^{+0.0297}$ & $1.0074_{-0.0035}^{+0.0144}$ & $0.7950_{+0.0377}^{-0.0498}$ & $0.9961_{-0.0007}^{+0.0083}$ & $1.0057_{-0.0024}^{+0.0081}$ \\
\hline K4V & $1.0139_{-0.0067}^{+0.0260}$ & $1.0115_{-0.0055}^{+0.0214}$ & $1.0057_{-0.0028}^{+0.0105}$ & $0.7955_{+0.0401}^{-0.0497}$ & $0.9916_{+0.0005}^{+0.0066}$ & $1.0051_{-0.0024}^{+0.0080}$ \\
\hline $\mathrm{K} 5 \mathrm{~V}$ & $1.0167_{-0.0075}^{+0.0354}$ & $1.0137_{-0.0062}^{+0.0288}$ & $1.0069_{-0.0031}^{+0.0142}$ & $0.8646_{+0.0351}^{-0.0337}$ & $0.9882_{+0.0013}^{+0.0068}$ & $1.0050_{-0.0029}^{+0.0083}$ \\
\hline K6V & $1.0136_{-0.0063}^{+0.0264}$ & $1.0110_{-0.0051}^{+0.0212}$ & $1.0055_{-0.0025}^{+0.0105}$ & $0.9473_{+0.0150}^{-0.0099}$ & $0.9850_{+0.0031}^{+0.0018}$ & $1.0014_{-0.0015}^{+0.0044}$ \\
\hline $\mathrm{K} 7 \mathrm{~V}$ & $1.0201_{-0.0113}^{+0.0349}$ & $1.0159_{-0.0089}^{+0.0274}$ & $1.0078_{-0.0044}^{+0.0132}$ & $0.9407_{+0.0116}^{-0.0122}$ & $0.9826_{+0.0024}^{+0.0034}$ & $0.9996_{-0.0009}^{+0.0057}$ \\
\hline K8V & $1.0153_{-0.0074}^{+0.0358}$ & $1.0120_{-0.0058}^{+0.0279}$ & $1.0058_{-0.0028}^{+0.0132}$ & $0.9406_{+0.0195}^{-0.0165}$ & $0.9813_{+0.0054}^{-0.0010}$ & $0.9980_{-0.0001}^{+0.0029}$ \\
\hline K9V & $1.0145_{-0.0073}^{+0.0276}$ & $1.0113_{-0.0057}^{+0.0213}$ & $1.0053_{-0.0027}^{+0.0099}$ & $0.9312_{+0.0147}^{-0.0250}$ & $0.9795_{+0.0039}^{-0.0033}$ & $0.9968_{+0.0003}^{+0.0022}$ \\
\hline
\end{tabular}

absolute transit depth changes that are notably smaller. This is owed to the opposing signals of unocculted spots and faculae that largely cancel out at visual wavelengths. At UV wavelengths, however, the effects of unocculted faculae dominate, and we find that the mean value of $\epsilon_{\mathrm{UV}}$ is 0.9900 , 0.9084 , and 0.8683 for $F, G$, and $\mathrm{K}$ dwarfs, respectively. In other words, unocculted faculae decrease transit depths in the UV, and the decreases are approximately $10 \%$ of the transit depth for $\mathrm{G}$ and $\mathrm{K}$ dwarfs on average.

Whether these effects are detectable will depend on both observational precisions and the planet-to-star radius ratio of the system in question, as the stellar contamination signal scales with the nominal transit depth. For comparison with observational precisions, we adopt $30 \mathrm{ppm}$ as our fiducial detection threshold. This is comparable to the typical transit depth uncertainty for current high-precision Hubble Space Telescope HST/WFC3 transmission spectrum observations (Kreidberg et al. 2014b) and systematic noise floors adopted by Greene et al. (2016) for NIRISS SOSS $(\lambda=1-2.5 \mu \mathrm{m}$; $20 \mathrm{ppm})$ and NIRCam grism $(\lambda=2.5-5.0 \mu \mathrm{m} ; 30 \mathrm{ppm})$ observations with the James Webb Space Telescope (JWST). For simplicity, we consider systems with a nominal transit depth of $D=1 \%$, which corresponds to giant planets with radii ranging from $R=0.4 R_{\text {Jup }}$ in the case of a K9V host star to $R=1.4 R_{\text {Jup }}$ in the case of an F9V host.

Under these assumptions, stellar contamination would produce a $30 \mathrm{ppm}$ feature and rise to the level of detectability when $\left|\epsilon_{\lambda}-1\right|>0.003$. Therefore, considering the mean values tabulated in Table 5, we find that for spots models, the effects of unocculted spots for typically active FGK stars are detectable in the UV and visual for spectral types G1V and later and in the NIR for spectral types G6V and later. For spots+faculae models, we find that the effects of unocculted heterogeneities are detectable in the UV for all spectral types F5V and later, while they are only detectable for $\mathrm{K} 3 \mathrm{~V}$ and later in the visual and $\mathrm{G} 4 \mathrm{~V}$ and later (excepting $\mathrm{K} 6 \mathrm{~V}$ and K7V) in the NIR.

To summarize, we find that unocculted heterogeneities in typically active $G$ and $K$ dwarfs can generally affect transmission spectra at levels relevant to current and nearfuture observational precisions. The effects are less pronounced for F dwarfs, though the impact of unocculted faculae may be apparent at UV wavelengths for these stars. While we focus on stars with typical activity levels here, we note that the stellar contamination signal obviously depends on the activity level of the star. Therefore, more active stars can produce larger stellar contamination signals than we detail here, and these may be detectable for earlier spectral types.

How the scale of the stellar contamination compares to that of planetary transmission features will depend on the parameters of the exoplanet in question. For the giant planets producing the nominal $D=1 \%$ transit depths that we consider here, planetary transmission features are considerably larger than the 30 ppm threshold that we adopt (e.g., Sing et al. 2016). Nonetheless, this analysis shows that for these planets, the stellar contamination signal of typically active FGK hosts can imprint on the transit depth at a scale that is detectable. Therefore, we conclude that potential stellar contamination should be a consideration for all high-precision transmission spectroscopy studies of FGK-hosted exoplanets, particularly 
for observations with later host stars, more active hosts, and at shorter wavelengths.

\subsection{Visual Slopes}

The most prominent feature of the contamination spectra from the spots models are the slopes produced at visual wavelengths. They are of particular interest here because they can be potentially confused with scattering slopes originating in exoplanet atmospheres.

To quantify the scale of the visual slopes in the contamination spectra, we first define the average value of $\epsilon_{\lambda}$ in a wavelength bin $\Delta \lambda$ centered on some wavelength $\lambda_{0}$ as

$$
\epsilon_{\mathrm{avg}}\left(\lambda_{0}, \Delta \lambda\right)=\frac{1}{\Delta \lambda} \int_{\lambda_{0}-\Delta \lambda / 2}^{\lambda_{0}+\Delta \lambda / 2} \epsilon_{\lambda} d \lambda .
$$

We then define the visual offset $\delta_{\text {vis }}$ as

$$
\delta_{\mathrm{vis}}=D\left[\epsilon_{\mathrm{avg}}\left(\lambda_{1}, \Delta \lambda\right)-\epsilon_{\mathrm{avg}}\left(\lambda_{2}, \Delta \lambda\right)\right],
$$

in which $D=1 \%, \quad \lambda_{1}=0.4 \mu \mathrm{m}, \quad \lambda_{2}=0.9 \mu \mathrm{m}, \quad$ and $\Delta \lambda=0.1 \mu \mathrm{m}$. Note that this formulation produces positive values for cases in which $\epsilon_{\lambda_{1}}>\epsilon_{\lambda_{2}}$, i.e., contamination spectra that increase toward shorter wavelengths. For each spectral type and model framework (spots and spots+faculae), we calculate $\delta_{\text {vis }}$ from the mean contamination spectrum. We also calculate $\delta_{\text {vis }}$ for the upper and lower $1 \sigma$ estimates for the contamination spectrum (i.e., the shaded regions in Figure 5), which we use to determine the $1 \sigma$ prediction interval on $\delta_{\text {vis }}$.

Figure 7 illustrates the visual offsets that we calculate for the spots and spotstfaculae models. The spots models produce positive visual offsets that increase in magnitude for later spectral types. For spectral types G9V and later, $\delta_{\text {vis }}$ is greater than the $30 \mathrm{ppm}$ detection threshold, meaning that unocculted spots on a typically active G9V-K9V host star can produce detectable increases in transit depths across the visual. However, these estimates are all consistent with the detection threshold at $1 \sigma$.

The spots+faculae models, on the other hand, produce visual offsets that are negative and increase in magnitude more starkly for later spectral types. We find that the absolute value of $\delta_{\text {vis }}$ is greater than the detection threshold at $1 \sigma$ confidence or higher for spectral types G0V and later. Faculae on K5V host stars have the largest effect, producing visual offsets of $\delta_{\text {vis }}=-350 \mathrm{ppm}$ at $3.4 \sigma$ confidence. These findings suggest that unocculted faculae can appreciably decrease visual transit depths in high-precision transmission spectra of exoplanets that orbit typically active $\mathrm{G}$ and $\mathrm{K}$ dwarfs.

This last point is interesting to consider in the context of the flat visual transmission spectra that are commonly observed for hot Jupiters (e.g., Gibson et al. 2013; Huitson et al. 2017; Parviainen et al. 2018). These are counter to model predictions for clear atmospheres, which should show transit depths that increase at shorter wavelengths as a signature of Rayleigh scattering (Seager \& Sasselov 2000; Fortney et al. 2010). Our results suggest that faculae can decrease visual transit depths at the level of a few hundreds of ppm, which is comparable to the precisions of current space-based (e.g., Sing et al. 2016) and ground-based observations (e.g., Nikolov et al. 2018; Espinoza et al. 2019). Therefore, it is possible that unocculted faculae could be counteracting signals from scattering slopes, making them at least in part responsible for the observed flat spectra. This observation underscores the importance of atmospheric

\section{Visual Offsets in FGK Transmission Spectra Assuming 1\% Transit Depth}
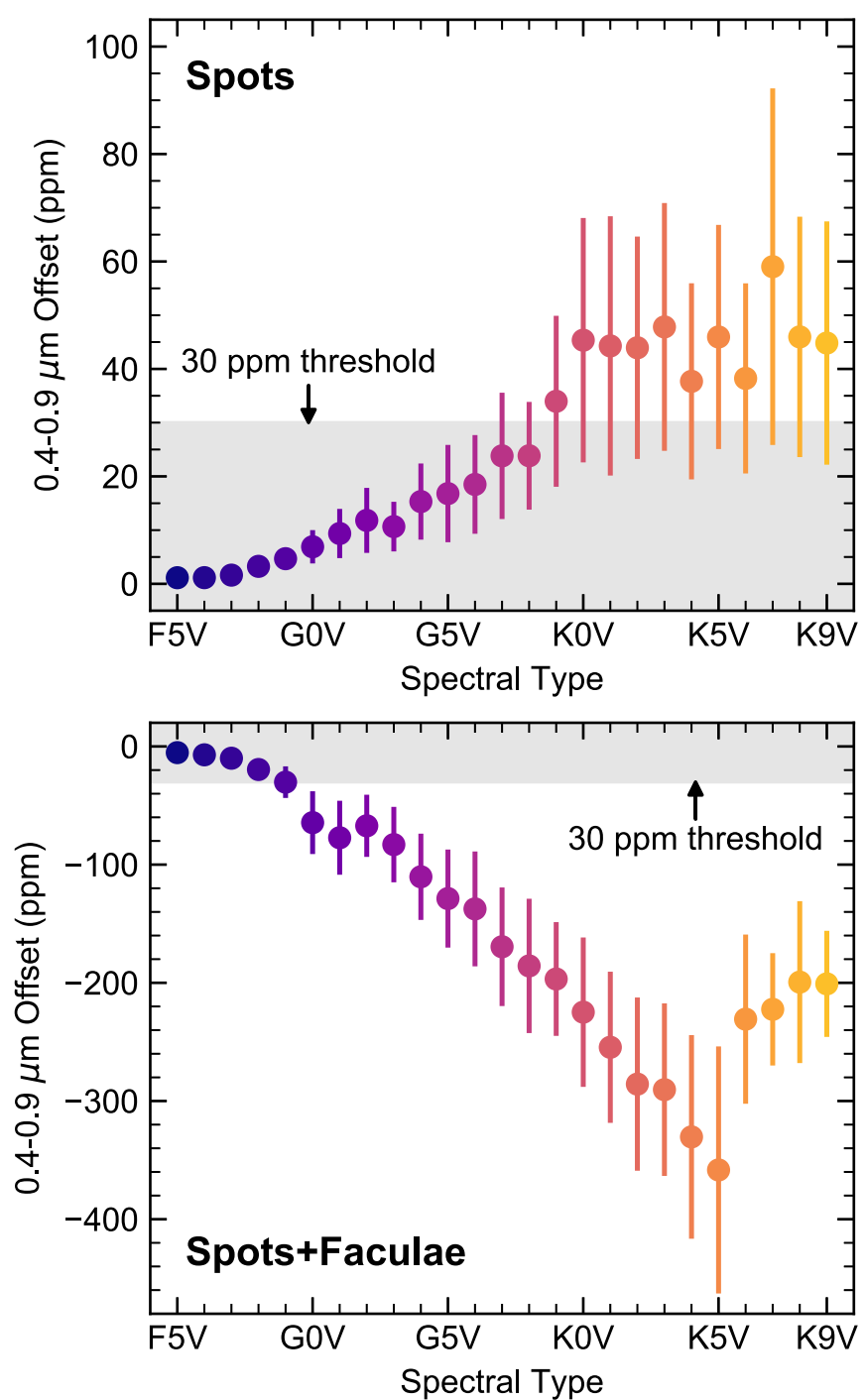

Figure 7. Visual offsets in transmission spectra for spots (top) and spots +faculae (bottom) models, assuming a nominal 1\% transit depth. For both model types, later spectral types produce larger visual offsets, but with opposing signs. The gray-shaded region illustrates offsets that are below our adopted $30 \mathrm{ppm}$ detection threshold. The data are color-coded by spectral type, following Figure 2. Note the varying $y$-axis scales.

retrievals that consider both stellar and planetary signals in transmission spectra (Pinhas et al. 2018; Espinoza et al. 2019), which are discussed further in Section 4.9.

On the other hand, the effect of unocculted faculae depends on the value of $f_{\text {fac }}$ for typically active $\mathrm{G}$ and $\mathrm{K}$ dwarfs, which we find could be between $\approx 8 \%$ for early-G dwarfs and $\approx 28 \%$ for late-K dwarfs (Table 4). While He I $10830 \AA$ equivalent width observations suggest that active $F$ and $G$ dwarfs can have active region filling factors of up to $\sim 80 \%-100 \%$ (Andretta et al. 2017), the Sun at solar maximum only reaches a maximum annual average value of $f_{\text {fac }} \approx 3 \%$ (Shapiro et al. 2014), a factor of a few lower than our estimates for early-G dwarfs and roughly an order of magnitude lower than our estimates for late-K dwarfs. Our approach relies on extrapolating the observed 10:1 facula-to-spot area ratio at solar maximum (Shapiro et al. 2014) to higher activity levels. 
Table 6

Vacuum Wavelengths Used in the Analysis of Transit Depth Line Offsets

\begin{tabular}{ll}
\hline \hline Feature & Wavelength(s) \\
\hline $\mathrm{Na}$ D doublet & 5894.570 \\
$\mathrm{H} \alpha$ & 6564.665 \\
$\mathrm{~K}$ I doublet & $7667.009,7701.084$ \\
\hline
\end{tabular}

However, this ratio may not hold for high activity levels generally. Additionally, other stars may exhibit different facula-to-spot area ratios than the Sun does. In this light, it possible that we have overestimated $f_{\text {fac }}$ and therefore the effects of faculae on transmission spectra. To complicate matters further, the Sun displays a time-dependence on the facula-to-spot area ratio throughout its activity cycle, with a facula-to-spot area ratio of 100:1 at solar minimum (Shapiro et al. 2016), primarily due to the absence of spots, and therefore we should expect other stars to do so as well. Future efforts to constrain facular coverages for interesting exoplanet host stars generally and in a time-resolved way near transit observations could be useful in this respect.

\subsection{Atomic Absorption Features}

The stellar contamination spectra for both spots and spots+faculae models show distinct features at narrow atomic lines in the visual. These include $\mathrm{H} \alpha$, the $\mathrm{Na} \mathrm{D}$ doublet, and the KI doublet, all of which also produce prominent features in transmission spectra of giant exoplanets. Broad absorption features from alkali metals point to cloud-free atmospheres and can be used to place constraints on their absolute abundances and in turn the atmospheric metallicity (Nikolov et al. 2018). On the other hand, increases in transit depth only around the narrow cores of these lines point to the presence of clouds and hazes (e.g., Sing et al. 2016). $\mathrm{H} \alpha$ absorption features can be used to probe column densities and excitation temperatures in exoplanetary exospheres (Jensen et al. 2012).

To quantify the effects of these features on observations, we define the transit depth line offset $\delta_{\text {line }}$ as

$$
\delta_{\text {line }}=D\left[\epsilon_{\text {avg }}\left(\lambda_{\text {line }}, \Delta \lambda\right)-\epsilon_{\text {cont }}\left(\lambda_{\text {line }}, \Delta \lambda\right)\right],
$$

in which the continuum value $\epsilon_{\text {cont }}$ is calculated as

$$
\begin{aligned}
\epsilon_{\mathrm{cont}}= & {\left[\epsilon_{\mathrm{avg}}\left(\lambda_{\text {line }}-1.5 \Delta \lambda, \Delta \lambda\right)\right.} \\
& \left.+\epsilon_{\mathrm{avg}}\left(\lambda_{\text {line }}+1.5 \Delta \lambda, \Delta \lambda\right)\right] / 2,
\end{aligned}
$$

and we set $D=1 \%$ and $\Delta \lambda=20 \AA$.

Table 6 lists the wavelengths used for the line offset analysis. We obtained air wavelengths for these features from the NIST Handbook of Basic Atomic Spectroscopic Data ${ }^{13}$ and converted them into vacuum wavelengths following Birch \& Downs (1994). The individual lines of the sodium doublet are separated by only $6 \AA$, so we used their average as the line wavelength. As the individual lines of the potassium doublet are separated by more than $30 \AA$, we calculated $\delta_{\text {line }}$ for each line separately and, finding them to be comparable, report the mean.

Figure 8 illustrates the line offsets that we calculate from the stellar contamination spectra and their $1 \sigma$ prediction intervals. None of the line offsets from the spots models register above

\footnotetext{
13 https://www.nist.gov/pml/handbook-basic-atomic-spectroscopic-data
}

our $30 \mathrm{ppm}$ detection threshold. For the spots+faculae models, we find that no $\mathrm{K}$ offsets are detectable and neither are $\mathrm{H} \alpha$ offsets, with the exception of an outlier at K2V. These lines are relatively narrow in the model stellar spectra, and thus the line offsets are relatively insignificant when integrated over bandpasses relevant to low-resolution transmission spectroscopy (i.e., $\Delta \lambda=20 \AA$ ). However, we find that $\mathrm{Na}$ offsets are detectable for spectral types G9V and later. Interestingly, $\mathrm{Na}$ offsets generally trend smoothly toward more negative values for spectral types F0V-K4V before sharply turning around and decreasing to near zero for late-K dwarfs. Inspection of the stellar component spectra shows that the $\mathrm{Na} \mathrm{D}$ doublet continuously broadens for spectral types from F0V to K9V. For the latest $\mathrm{K}$ dwarfs, the $\mathrm{Na} \mathrm{D}$ doublet becomes broader than $20 \AA$, which complicates the determination of the continuum level. Therefore, the turn-around in the $\mathrm{Na}$ offset seen for the latest $\mathrm{K}$ dwarfs is an artifact of our selection for $\Delta \lambda$ and not representative of a physical transition in the stellar atmospheres.

The upshot of this analysis is that unocculted spots on typically active FGK dwarfs are not likely to produce detectable changes in transit depths around atomic features, though unocculted faculae can alter transit depths detectably around the $\mathrm{Na} \mathrm{D}$ doublet. However, the caveats discussed in Section 4.2 regarding our prescriptions for modeling faculae apply here as well.

\subsection{Trends in Visual Features}

The analysis in the previous sections shows that with a few exceptions, visual stellar contamination features are generally not detectable in transmission spectra of exoplanets hosted by typically active FGK dwarfs. However, more active host stars may still be problematic. To investigate this, we repeated the analysis presented in Section 3, defining an "active star" as one showing a rotational variability amplitude in the Kepler bandpass equal to the $84 \%$ percentile for its spectral type. Accordingly, we use as the reference amplitudes for this "active case" the $1 \sigma$ upper limits on the variability amplitudes (i.e., the $84 \%$ percentiles) from Table 3 . In this case, the active region covering fractions that correspond to the reference amplitude are a factor of a few higher than for the nominal case. Specifically, in the spots case, $f_{\text {spot }}$ is seven times larger on average, while in the spots+faculae case, $f_{\text {spot }}$ and $f_{\text {fac }}$ are four and three times larger, respectively.

These larger covering fractions produce larger stellar contamination signals, making more features detectable above our adopted threshold. In general, the offsets trend with spectral type in the same manner as shown in Figures 7 and 8, but the scales of the offsets are exaggerated. For the spots models, positive visual offsets are larger than $30 \mathrm{ppm}$ for spectral types F9V and later and reach a peak of $254 \mathrm{ppm}$ at spectral type $\mathrm{K} 5 \mathrm{~V}$. Additionally, positive $\mathrm{Na}$ offsets are $>30 \mathrm{ppm}$ for spectral types G4V-K2V. For the spots+faculae models, the negative visual offsets are $>30 \mathrm{ppm}$ in magnitude for all spectral types and reach a peak value of -483 at spectral type $\mathrm{K} 5 \mathrm{~V}$. Positive $\mathrm{H} \alpha$ offsets and negative $\mathrm{Na}$ offsets are detectable for late-G to mid-K dwarfs. $\mathrm{K}$ offsets are smaller than our adopted threshold for all spectral types, but begin to increase for late-K dwarfs.

The magnitudes of these offsets, particularly with respect to the visual slope and the $\mathrm{Na}$ line offset, are such that they could be confused with features originating the the atmospheres of 


\section{Transit Depth Line Offsets in FGK Transmission Spectra Assuming 1\% Transit Depth}
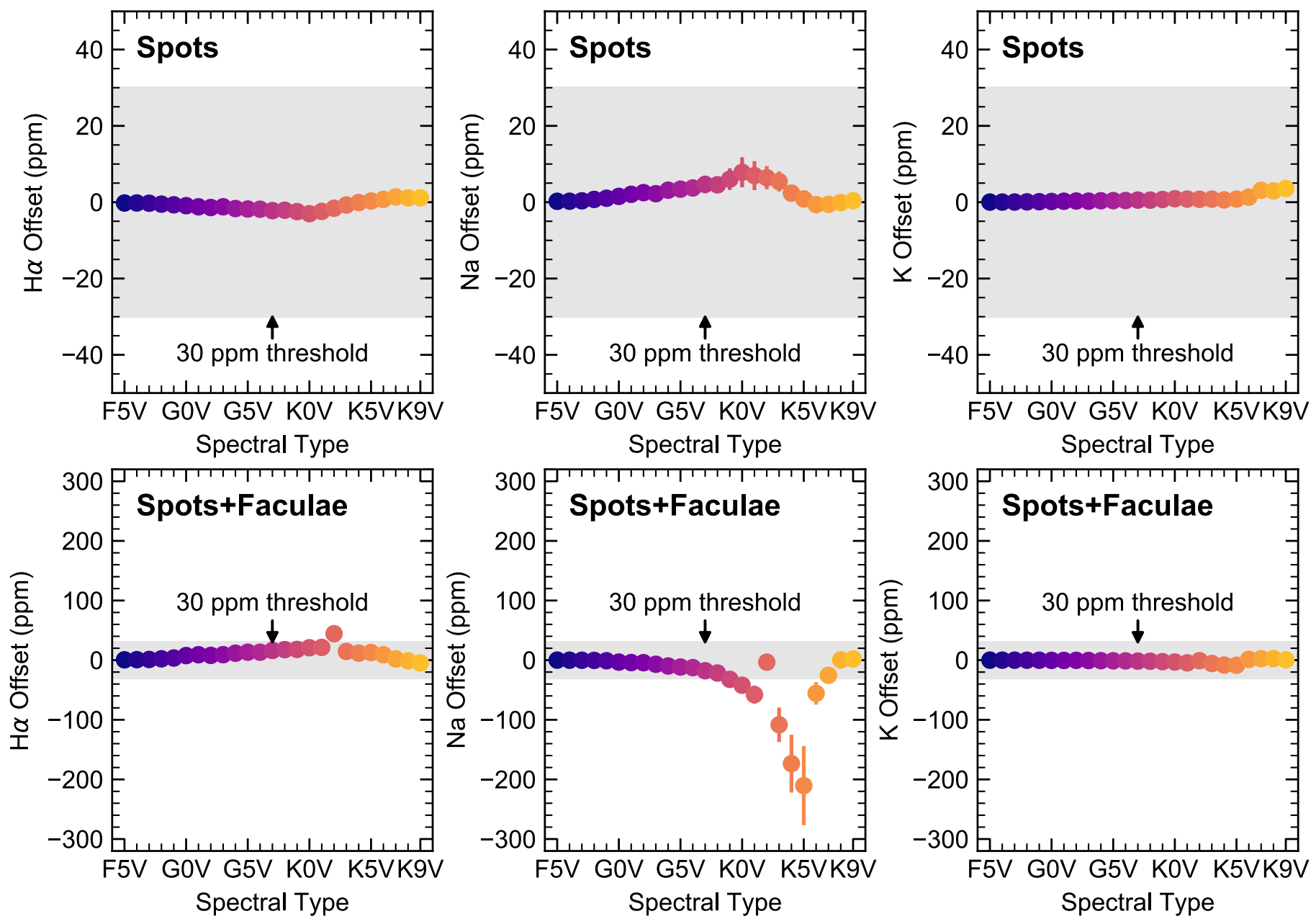

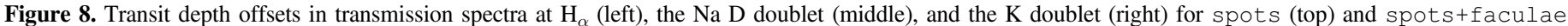

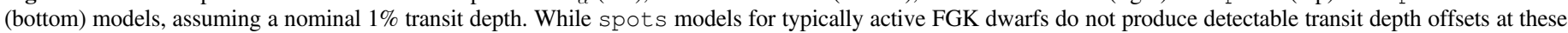

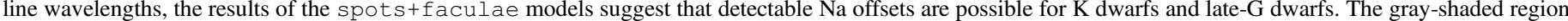

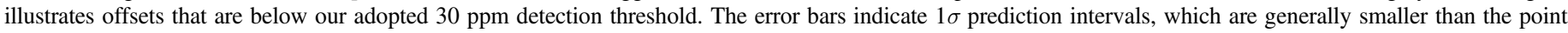
size. The data are color-coded by spectral type, following Figure 2. Note the varying $y$-axis scales.

transiting exoplanets. However, these features trend with each other and with spectral type in systematic ways. These trends can be used to identify features with a stellar origin and distinguish them from planetary ones.

Figure 9 illustrates these trends for the spots models in the active case. Generally, all offsets are near-zero for the earliest spectral type and increase for later spectral types. The largest offset overall is the visual $(0.4-0.9 \mu \mathrm{m})$ offset, and the largest line offset is that of Na. Starting with F5V, these both increase for later spectral types, reaching maxima around late-G dwarfs, after which the visual offsets remain roughly the same, while the Na offsets decrease. The signs and relative magnitude of these features could point to a stellar origin for features observed in transmission spectra, particularly for exoplanets hosted by late-G or K dwarfs.

Figure 10 illustrates the observed trends in offsets for the spots+faculae models. Compared to the spots models, the offsets have larger magnitudes and the trends have the opposite signs because unocculted faculae can dominate the visual slope and line offsets.

In general, identifying these trends in observations remains a challenge, given the currently reachable precision. Of the trends illustrated in Figures 9 and 10, only that between the $\mathrm{Na}$ line offset and the visual slope in Figure 10 produces changes in both features well above the adopted $30 \mathrm{ppm}$ detection threshold (and, essentially, only for $\mathrm{K}$ dwarfs). This points to a potential limitation of the usefulness of these diagnostics. However, these trends will be more evident in stars that are more active than our "active case" (recalling that we define an "active star" as one with a rotational variability only $1 \sigma$ above the median), and future observational precisions may very well push below the $30 \mathrm{ppm}$ threshold. For these reasons, we point out these trends so that they may be of use in distinguishing stellar and planetary features in transmission spectra in future studies.

\subsection{Molecular Absorption Features}

In addition to enabling studies of atomic absorption features, transmission spectra are useful probes of molecular absorption bands in exoplanet atmospheres. The contamination spectra plotted in Figure 5 show broad features owing to changes in molecular opacities between the immaculate photosphere and stellar active regions. Figure 5 also illustrates wavelengths of 


\section{Visual Features in FGK Transmission Spectra Due to Unocculted Spots}

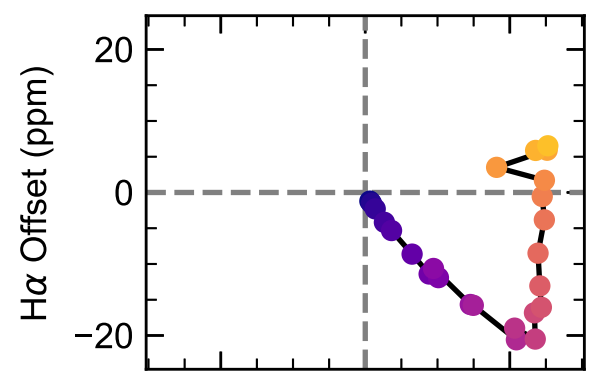

Assuming 1\% Transit Depth
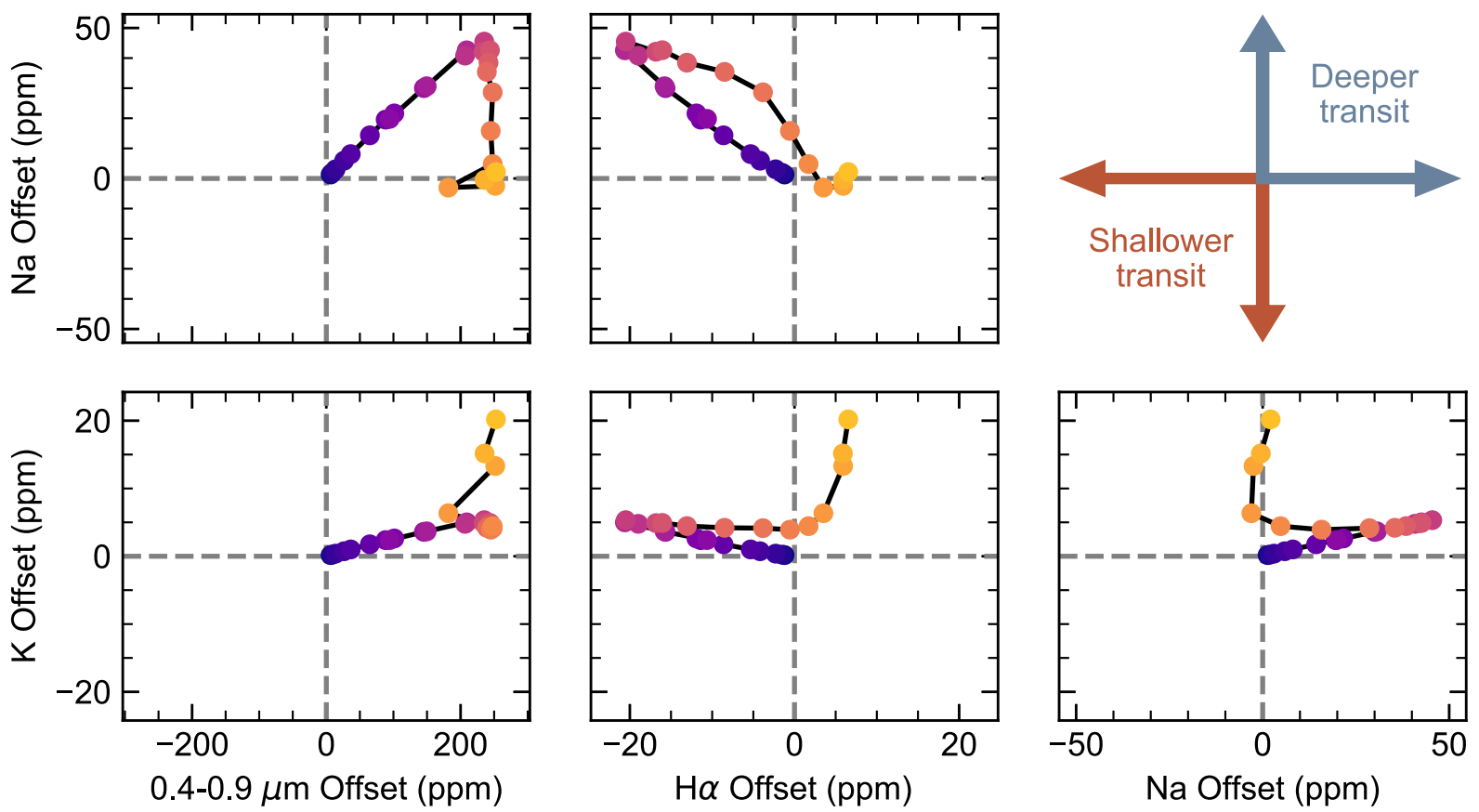

F5V

GOV

$\mathrm{K} 5 \mathrm{~V}$

G5V

$\mathrm{KOV}$ के

Figure 9. Trends in transit depth changes in visual FGK contamination spectra features for spots models. The magnitudes of stellar contamination features for FGK dwarfs generally grow with later spectral types. They also trend in systematic ways in terms of their signs and relative strengths, which we suggest could be used to identify features with a stellar origin. Positive (negative) values indicate deeper (shallower) transits. Note the varying axis scales.

interest for some potentially detectable molecules in exoplanet atmospheres, including $\mathrm{CH}_{4}, \mathrm{CO}, \mathrm{CO}_{2}, \mathrm{H}_{2} \mathrm{O}, \mathrm{N}_{2} \mathrm{O}, \mathrm{O}_{2}$, and $\mathrm{O}_{3}$. If the values of stellar contamination spectra within these bands differ systematically from those of adjacent wavelengths, the stellar signal could mimic or mask exoplanetary molecular features in transmission spectra.

We investigate this possibility quantitatively following a similar approach to the analysis of atomic absorption features detailed in Section 4.3. We define the transit depth band offset as

$$
\delta_{\text {band }}=D\left[\epsilon_{\text {avg }}\left(\lambda_{\text {band }}, \Delta \lambda_{\text {band }}\right)-\epsilon_{\text {cont }}\left(\lambda_{\text {line }}, \Delta \lambda_{\text {band }}, \Delta \lambda\right)\right],
$$

in which $\lambda_{\text {band }}$ is the central wavelength of the molecular band, $\Delta \lambda_{\text {band }}$ is its width, and we set $D=1 \%$ as before. In this case, the continuum value $\epsilon_{\mathrm{cont}}$ is calculated as

$$
\epsilon_{\mathrm{cont}}=\left[\epsilon_{\mathrm{avg}}\left(\lambda_{0}, \Delta \lambda\right)+\epsilon_{\mathrm{avg}}\left(\lambda_{1}, \Delta \lambda\right)\right] / 2,
$$

in which $\quad \lambda_{0}=\lambda_{\text {band }}-\Delta \lambda_{\text {band }}-0.5 \Delta \lambda$, $\lambda_{1}=\lambda_{\text {band }}+\Delta \lambda_{\text {band }}+0.5 \Delta \lambda$, and we set $\Delta \lambda=0.1 \mu \mathrm{m}$. Thus, $\delta_{\text {band }}$ represents the difference in the average value of a transmission spectrum within a molecular absorption band relative to the average of the flanking regions. We determine the value of $\delta_{\text {band }}$ for each of the bands ${ }^{14}$ illustrated in Figure 5 and calculate the molecular offset $\delta_{\text {mol }}$ for each molecule as the average of $\delta_{\text {band }}$ for the molecular bands weighted by the bandwidths.

We find that none of the molecular offsets for $\mathrm{CH}_{4}, \mathrm{CO}$, $\mathrm{CO}_{2}, \mathrm{H}_{2} \mathrm{O}, \mathrm{N}_{2} \mathrm{O}, \mathrm{O}_{2}$, or $\mathrm{O}_{3}$ are larger than our adopted $30 \mathrm{ppm}$ detection threshold. The largest offsets are those for $\mathrm{O}_{2}, \mathrm{H}_{2} \mathrm{O}$, and $\mathrm{CH}_{4}$, which are illustrated in Figure 11. While all are still below the adopted detection threshold, the later spectral types produce relatively larger offsets. For the spots models, the $\mathrm{O}_{2}$ and $\mathrm{H}_{2} \mathrm{O}$ offsets are positive, while the $\mathrm{CH}_{4}$ offsets are negative. The offsets trend similarly in the spots+faculae models, except that the $\mathrm{O}_{2}$ and $\mathrm{H}_{2} \mathrm{O}$ offsets start to become more negative for spectral types later than around $\mathrm{K} 5 \mathrm{~V}$, while those for $\mathrm{CH}_{4}$ become more positive. In each case, $\mathrm{O}_{2}$ and $\mathrm{H}_{2} \mathrm{O}$ offsets trend in the opposite direction as the $\mathrm{CH}_{4}$ offsets.

Of course, these offsets are calculated for typically active FGK dwarfs and nominal transit depths of $1 \%$, so more active

\footnotetext{
The longest-wavelength bands of $\mathrm{H}_{2} \mathrm{O}$ and $\mathrm{O}_{3}$ are within $0.1 \mu \mathrm{m}$ of the long-wavelength end of the contamination spectra, so these two bands have truncated baselines for determining $\epsilon_{\text {cont }}$.
} 


\section{Visual Features in FGK Transmission Spectra Due to Unocculted Spots+Faculae Assuming 1\% Transit Depth}
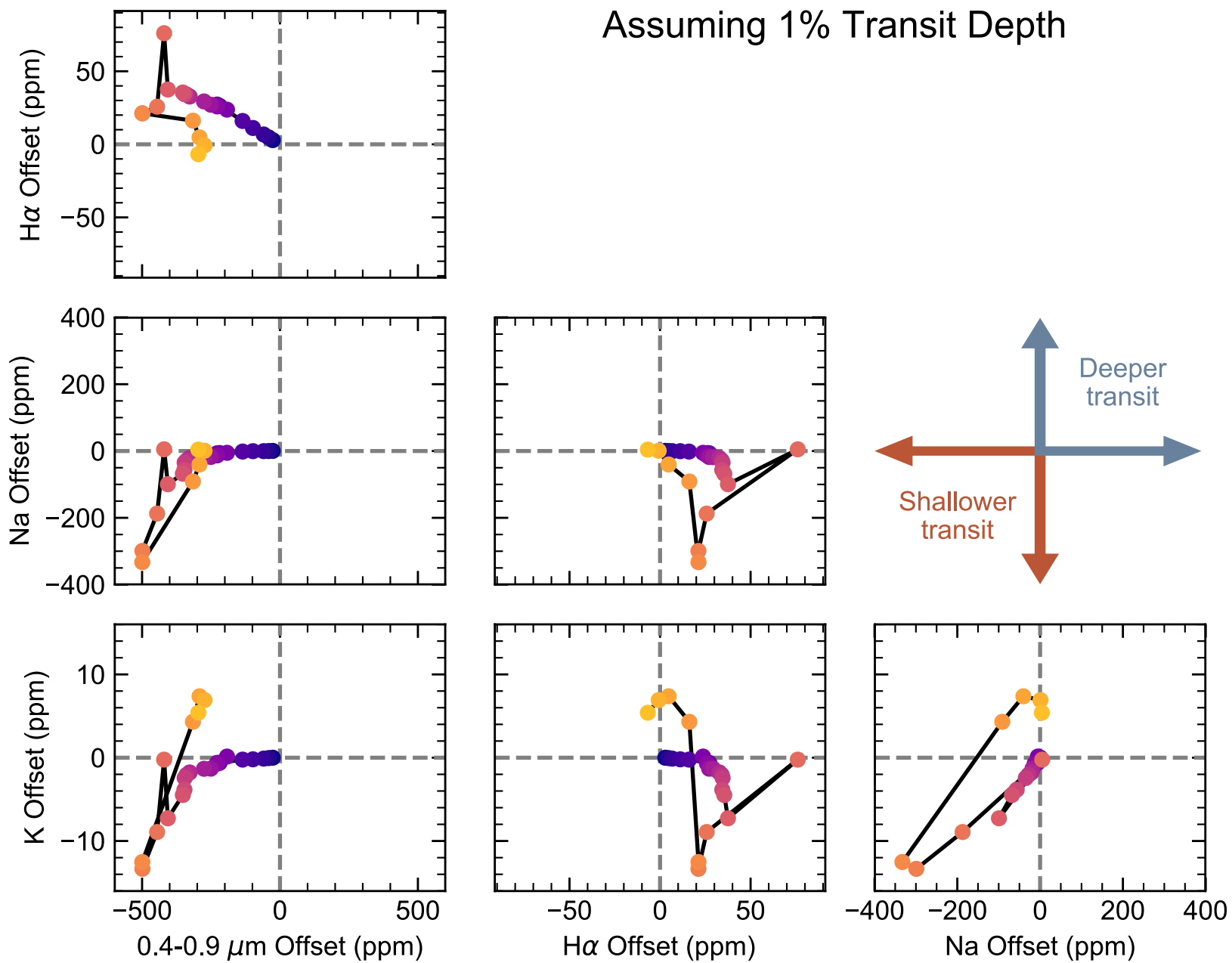

F5V

GOV

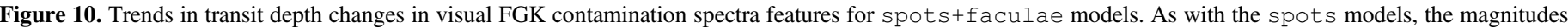

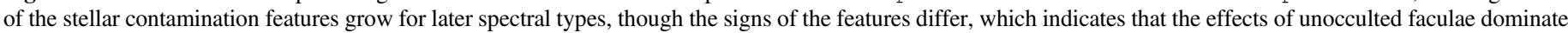
at visual wavelengths. Positive (negative) values indicate deeper (shallower) transits. Note the varying axis scales.

host stars or deeper transit depths could render the molecular offsets larger than our adopted detection threshold. By the same token, improvements in observational techniques or instrumentation could enable finer precisions in transmission spectra than $30 \mathrm{ppm}$. In any case, we point out here the trends in these molecular offsets so that they may be useful for identifying stellar contamination features in transmission spectra in future studies.

\subsubsection{Water Spectral Features}

Water features in transmission spectra are of particular interest, given their ubiquity in existing hot Jupiter (e.g., Sing et al. 2016) and some hot super-Neptune (Fraine et al. 2014; Stevenson et al. 2016; Wakeford et al. 2017) observations to date. For typically active FGK dwarfs, considering the spots models, we find the largest offsets at $\mathrm{H}_{2} \mathrm{O}$ absorption bands for spectral type $\mathrm{K} 7 \mathrm{~V}$. In this case, unocculted spots inflate a $1 \%$ transit depth by $\delta_{\mathrm{H}_{2} \mathrm{O}}=7_{-4}^{+11} \mathrm{ppm}$. For spots+faculae models, by comparison, the largest offset, $\delta_{\mathrm{H}_{2} \mathrm{O}}=9_{-3}^{+8} \mathrm{ppm}$, is found for $\mathrm{K} 4$ dwarfs. Generalized for any transit depth, these values correspond to maximal values of $\epsilon_{\mathrm{H}_{2} \mathrm{O}}=1.0007_{-0.0004}^{+0.0011}$ for spots models and $\epsilon_{\mathrm{H}_{2} \mathrm{O}}=1.0009_{-0.0003}^{+0.0008}$ for spots +faculae models.

In both cases, the net effect of unocculted heterogeneities is to increase transit depths, potentially mimicking a planetary water absorption feature. The scale of the effect, however, is far smaller than that of planetary features that have been probed in transmission spectra to date. For comparison, the commonly studied $1.4 \mu \mathrm{m}$ water absorption band has an amplitude of a few hundreds of ppm for hot Jupiters (e.g., Sing et al. 2016) and the hot Neptunes in which it has been detected so far (Fraine et al. 2014; Wakeford et al. 2017). Furthermore, the observed stellar contamination signal scales with the nominal transit depth (following Equation (6)), so for transits of planets smaller than hot Jupiters and Neptunes - in which the planetary atmospheric signals will be smaller than the existing detections - the stellar contamination signal will be correspondingly smaller as well. Thus, we conclude that stellar contamination at wavelengths of interest for $\mathrm{H}_{2} \mathrm{O}-$ or $\mathrm{CH}_{4}, \mathrm{CO}, \mathrm{CO}_{2}, \mathrm{~N}_{2} \mathrm{O}, \mathrm{O}_{2}$, or $\mathrm{O}_{3}$, for that matter-is not problematic for transmission spectroscopy studies involving typically active FGK host stars. For a discussion of these species in transmission spectra of Earth-like planets, see Section 4.6. As always, stellar activity is an important caveat: special care should be taken in studies 


\section{Molecular Offsets in FGK Transmission Spectra Assuming 1\% Transit Depth}
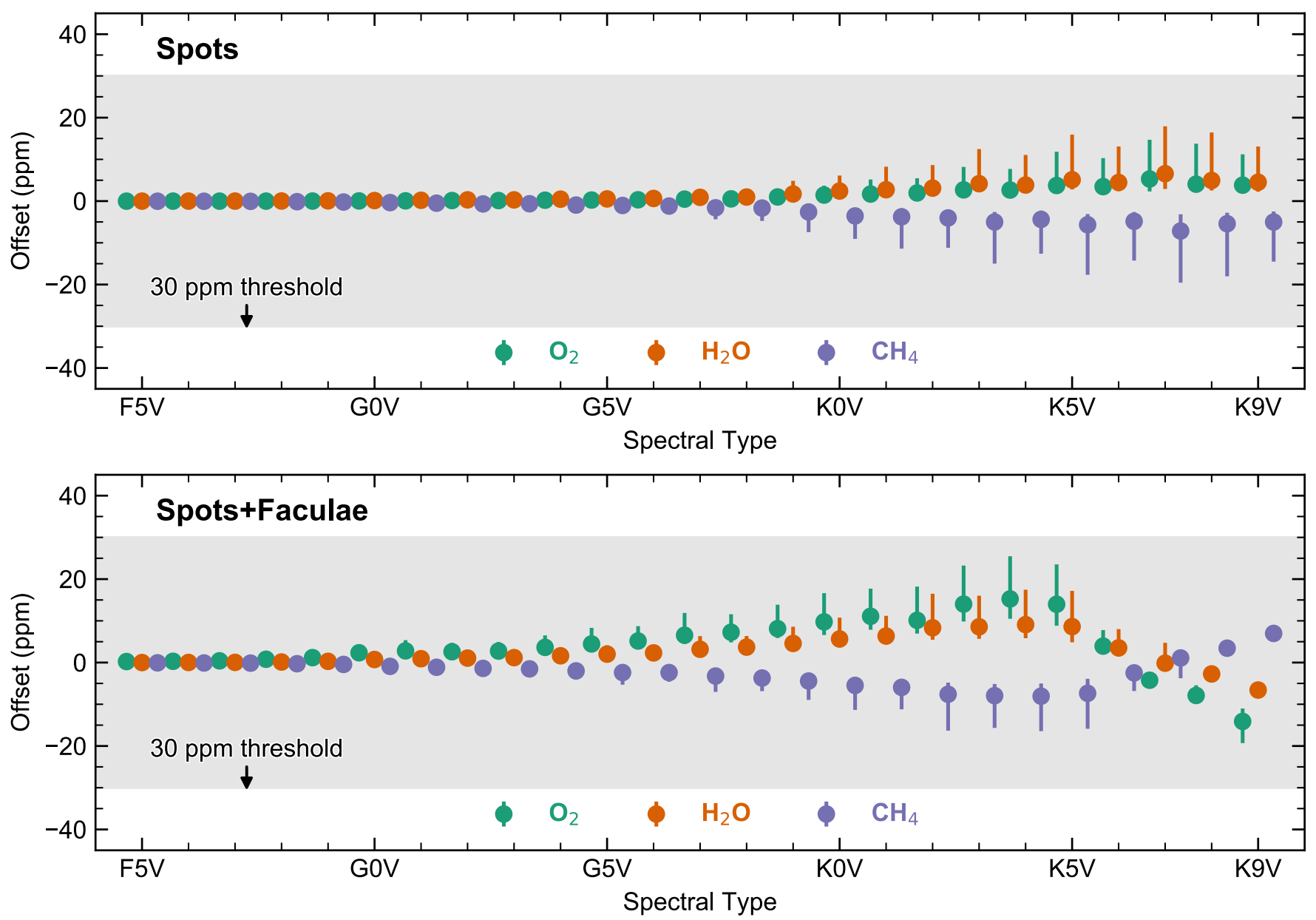

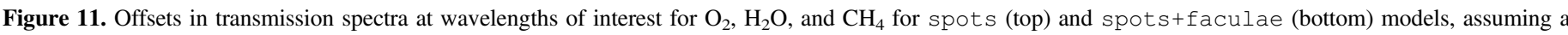

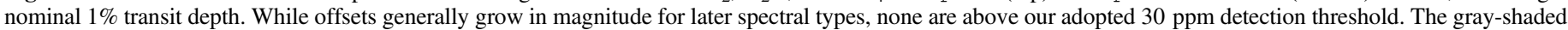
region illustrates offsets that are below our adopted $30 \mathrm{ppm}$ detection threshold.

involving host stars with larger variability amplitudes than the medians tabulated in Table 3 or other indicators of stellar activity.

Another important caveat comes from a potential limitation of our approach, which is that we fix spot and facula temperatures to set values. As we are investigating an already large parameter space, out of necessity, we do not allow for a range of active region temperatures for a given spectral type. However, a range of active region temperatures is likely present on a given star. On the Sun $\left(T_{\text {eff }}=5800 \mathrm{~K}\right)$, for example, sunspot umbrae generally have temperatures of 3900-4800 K and penumbrae 5400-5550 K (Solanki 2003, and references therein). In this study we adopt $T_{\text {spot }}=4030 \mathrm{~K}$ for G2 dwarfs, roughly in line with these values. Nonetheless, sunspots as cool as $T_{\text {spot }} \simeq 3200 \mathrm{~K}$ have been observed. These are notable because water forms in sunspots cooler than about $3900 \mathrm{~K}$ and represents the dominant opacity source in unusually cool sunspots (Wallace et al. 1995). Therefore, adopting a fixed spot temperature may lead us to underestimate $\delta_{\mathrm{H}_{2} \mathrm{O}}$ for spectral types G8V and earlier, for which we set $T_{\text {spot }}>3900 \mathrm{~K}$. Still, the values of $\delta_{\mathrm{H}_{2} \mathrm{O}}$ that we determine for spectral types $\mathrm{G} 9 \mathrm{~V}-$ $\mathrm{K} 9 \mathrm{~V}$ are roughly two orders of magnitude below the amplitudes of planetary water absorption features that have been detected to date. This fact suggests that our top-level conclusions are likely not affected by fixing active region temperatures to set values, though we caution that more detailed investigations are warranted for specific observational cases in which the host star is relatively active or the expected scale of the planetary feature is smaller than in the existing detections.

\subsection{Earth-Sun Analog Systems}

One interesting example that warrants further investigation here is that of Earth-Sun analog systems. These systems are targets of long-term efforts to characterize truly Earth-like exoplanets and search for biosignatures. Given the Earth-Sun radius ratio, the nominal transit depth of such a system is $D_{\oplus}=84 \mathrm{ppm}$. Within the wavelength range of this study, Earth's transmission spectrum displays prominent absorption bands from from $\mathrm{H}_{2} \mathrm{O}, \mathrm{CO}_{2}, \mathrm{O}_{2}$, and $\mathrm{O}_{3}$ (e.g., Ehrenreich et al. 2006; Kaltenegger \& Traub 2009; Pallé et al. 2009). An order of magnitude approximation for the scale of spectral features in transmission spectra $\Delta D$ (Miller-Ricci et al. 2009) for an EarthSun system gives

$$
\Delta D_{\oplus} \sim \frac{2 H_{\oplus} R_{\oplus}}{R_{\odot}^{2}}=2 \times 10^{-7}
$$




\section{Molecular Offsets for Earth-Sun Analog}
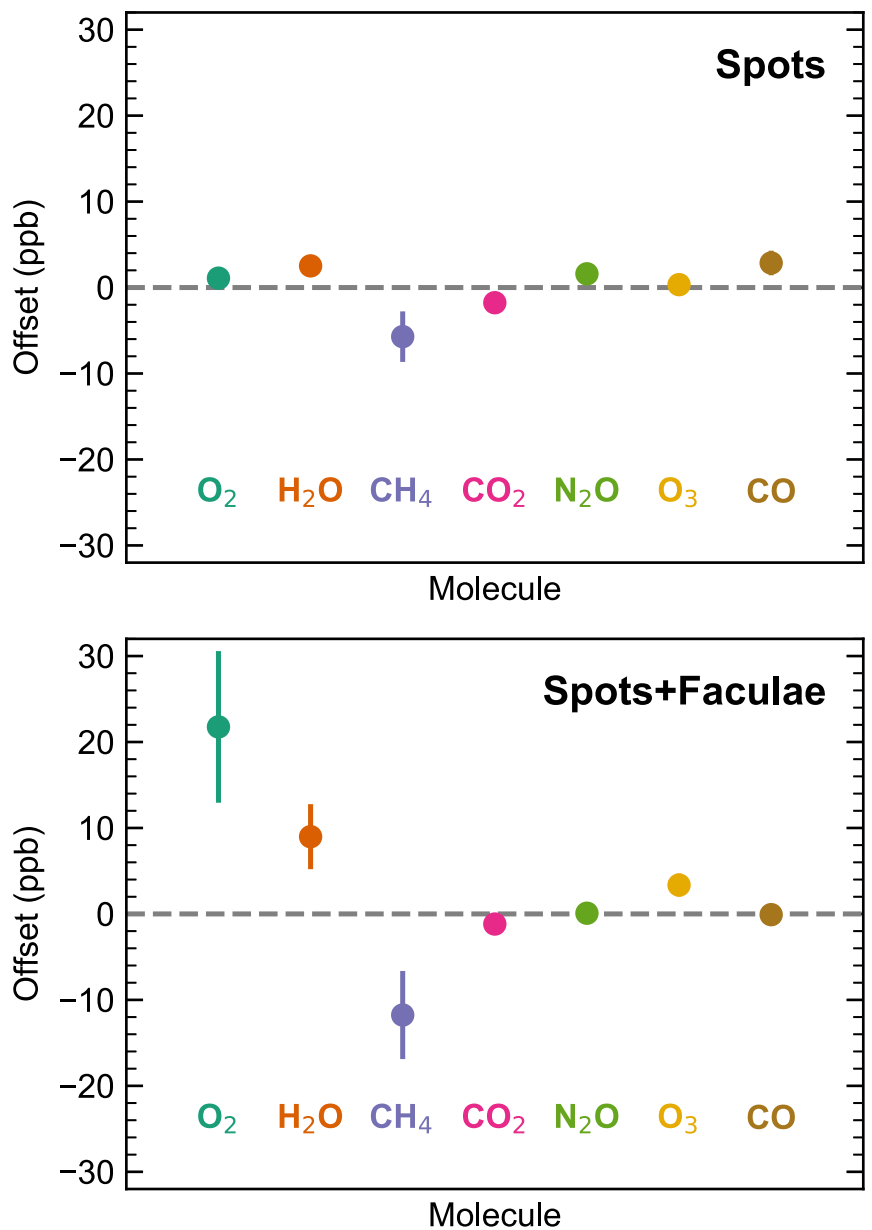

Figure 12. Transit depth offsets within molecular absorption bands in transmission spectra of a typically active G2V dwarf, assuming a nominal $84 \mathrm{ppm}$ transit depth. For both spots (top) and spots +faculae (bottom) models, the offsets at wavelengths of interest for important planetary atmospheric species are more than an order of magnitude smaller than $\Delta D_{\oplus}$. The molecules are ordered by the the wavelength of their respective shortestwavelength bands (see Figure 5), which illustrates that the largest offsets are generally found for the molecules with bands at shorter wavelengths. The error bars indicate $1 \sigma$ prediction intervals, which are generally smaller than the point size. Note that the offset values are given in $\mathrm{ppb}$.

or 200 parts-per-billion (ppb) for features covering a single scale height.

For comparison, Figure 12 illustrates the molecular offsets for important planetary molecular absorbers in the $0.05-5.5 \mu \mathrm{m}$ range in an Earth-Sun analog system. To calculate these offsets, we use the stellar contamination spectrum for the typically active G2 dwarf (presented in Figure 5) and assume $D=84 \mathrm{ppm}$. Of the molecular features highlighted in Figure 5, we find the largest overall offset, $\sim 20 \mathrm{ppb}$, for $\mathrm{O}_{2}$ with the spotstfaculae model. The remaining offsets are generally $<10 \mathrm{ppb}$. In other words, the scale of the stellar contamination is roughly an order of magnitude smaller than a single-scale-height planetary transmission feature.

We conclude, therefore, that stellar contamination in EarthSun analog systems will not preclude low-resolution observations of planetary molecular features. High-resolution $(R \sim 100,000)$ observations, in which planetary lines are Doppler-shifted away from stellar lines (e.g., Snellen et al.
2010; Brogi et al. 2012; Rodler et al. 2012), should suffer even less from this effect. Given the future potential for highresolution observations, including searches for potential biosignatures (Snellen et al. 2013; Rodler \& López-Morales 2014; Ben-Ami et al. 2018), a detailed examination of the effect of stellar contamination on high-resolution observations of Earth-Sun analog systems would be worthwhile, but is beyond of the scope of this work. In any case, the minute scales of both $\Delta D_{\oplus}$ and $\delta_{\text {mol }}$ emphasize the importance of precisely understanding the photospheric properties of interesting exoplanet host stars, including active region contrasts and covering fractions at the time of transit observations.

\subsection{TiO/VO in Visual Contamination Spectra}

Titanium oxide (TiO) and vanadium oxide (VO) are two important molecular absorbers in planetary atmospheres, particularly in those of hot giant planets. They display significant opacity across the full visual wavelength range (Hill et al. 2013), which allows them to significantly affect pressuretemperature profiles of hot giant planets. Evidence for $\mathrm{TiO} / \mathrm{VO}$ absorption features in the transmission spectra of the ultra-hot Jupiter WASP-121b (Evans et al. 2016), for example, pointed to the presence of a thermal inversion in the planetary atmosphere, which was later confirmed by a thermal emission spectrum obtained through secondary-eclipse observations (Evans et al. 2017).

At the same time, $\mathrm{TiO} / \mathrm{VO}$ are also present in stellar atmospheres. They absorb more strongly at cooler stellar temperatures, and observations of $\mathrm{TiO} / \mathrm{VO}$ molecular features have long been used to constrain spot temperatures and filling factors (Vogt 1979, 1981; Ramsey \& Nations 1980). In this study, we find that unocculted spots can impart $\mathrm{TiO} / \mathrm{VO}$ features in exoplanet transmission spectra. This is most clearly illustrated by the K-dwarf contamination spectra in the lower left panel of Figure 6, which closely resemble the absorption spectrum of TiO (Hill et al. 2013).

A straightforward calculation of $\delta_{\mathrm{mol}}$ for $\mathrm{TiO}$ and $\mathrm{VO}$ as defined in Section 4.5 is complicated by the tight packing of molecular bands across the visual wavelength range, where their absorption cross-sections are important. However, we can gain some quantitative insight into the impact of strong visual molecular absorbers in spots on transmission spectra by investigating deviations from simple slopes in the visual contamination spectra. To this end, we define the $\mathrm{TiO} / \mathrm{VO}$ offset as

$$
\delta_{\mathrm{TiO} / \mathrm{VO}}=\max \left[D\left(\epsilon-\epsilon_{\text {line }}\right)\right],
$$

in which $\epsilon$ is the stellar contamination spectrum in the $0.4-0.9 \mu \mathrm{m}$ range, $\epsilon_{\text {line }}$ is a simple line fit to the points used in Section 4.2 to define the visual offset (Equation (8)), and we set $D=1 \%$ as before. In other words, $\delta_{\mathrm{TiO} / \mathrm{vO}}$ provides an estimate of the amplitude of the deviations from a simple slope in a visual stellar contamination spectrum for a planet with a $1 \%$ transit depth. To simulate observational precisions, we calculate $\delta_{\mathrm{TiO} / \mathrm{vO}}$ with stellar contamination spectra that have been down-sampled from the resolution of the PHOENIX models to a spectral resolution of $100 \AA$.

We calculate $\delta_{\mathrm{TiO} / \mathrm{VO}}$ for all spots models, in which visual molecular features are most apparent, including contamination spectra and their $1 \sigma$ prediction intervals for our nominals case and the active case defined in Section 4.4. The results are 


\section{TiO/VO Features in FGK Transmission Spectra} Assuming 1\% Transit Depth
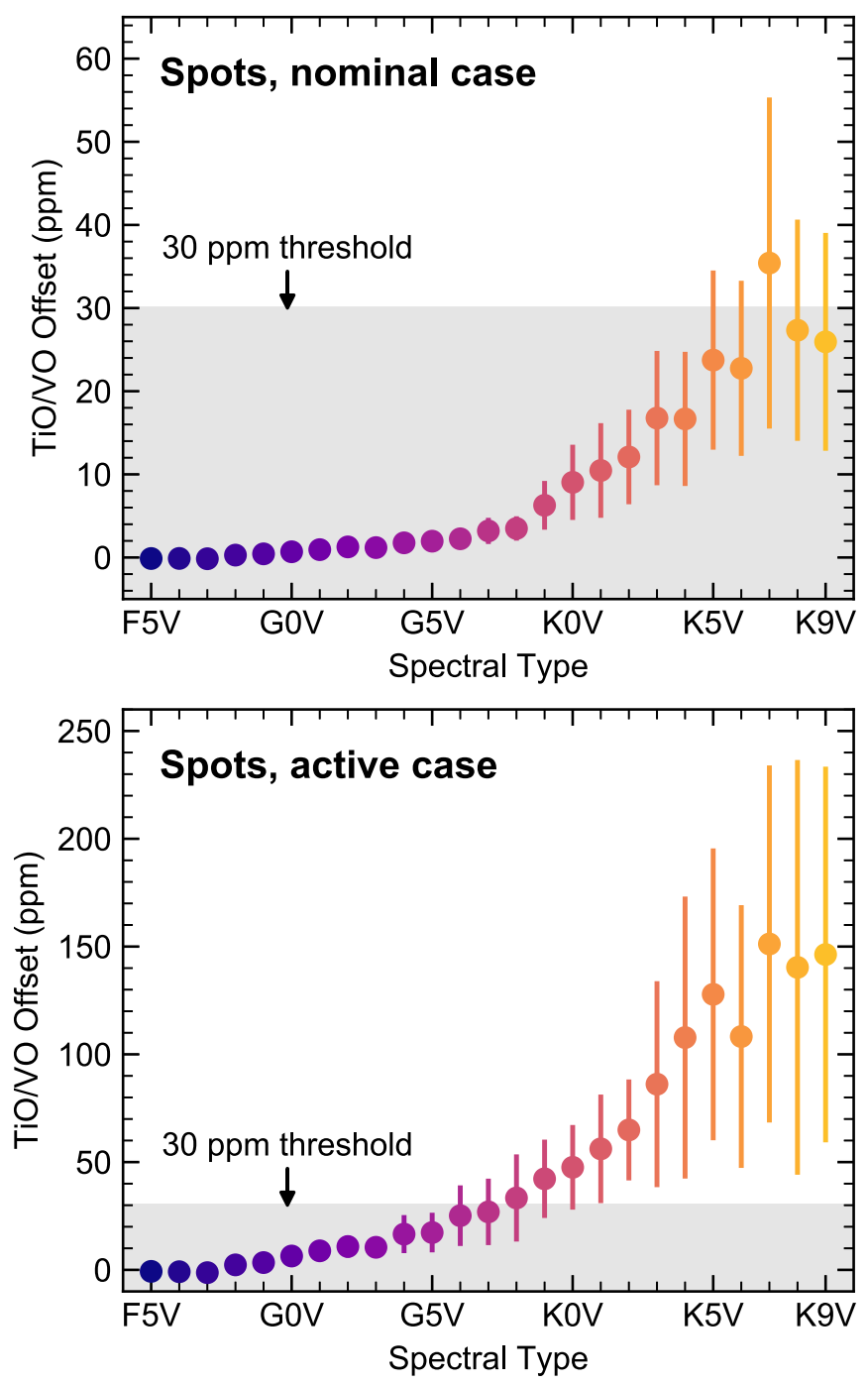

Figure 13. $\mathrm{TiO} / \mathrm{VO}$ offsets in transmission spectra for spots models, assuming a nominal $1 \%$ transit depth. The scale of the offsets grows with later spectral types. For typically active FGK dwarfs (top), offsets are relatively small, reaching a few tens of ppm for late-K dwarfs. For more-active FGK dwarfs (bottom, see Section 4.4), offsets are roughly $150 \mathrm{ppm}$ for late-K dwarfs. The gray-shaded region illustrates offsets that are below our adopted $30 \mathrm{ppm}$ detection threshold. The data are color-coded by spectral type, following Figure 2. Note the varying $y$-axis scales.

illustrated in Figure 13. The offsets grow with later spectral types. For our nominal case of typically active FGK dwarfs, we find that only K7 dwarfs produce offsets greater than our adopted $30 \mathrm{ppm}$ detection threshold, though none of the estimates of $\delta_{\mathrm{TiO} / \mathrm{VO}}$ are greater than a few tens of ppm. For our active case, on the other hand, we find estimates for $\delta_{\mathrm{TiO} / \mathrm{VO}}$ that are greater than $30 \mathrm{ppm}$ for spectral types G8V and later and that are roughly $150 \mathrm{ppm}$ for late-K dwarfs. We conclude that visual molecular features are generally not significant for typically active FGK dwarfs, though they can be significant for more-active $\mathrm{K}$ and late- $\mathrm{G}$ dwarfs. Therefore, we caution that stellar molecular features should be a consideration for late-G and $\mathrm{K}$ dwarfs, especially if they display larger variability amplitudes than the medians tabulated in Table 3 or other indications of stellar activity. Examples of such systems include WASP-6, WASP-19, and HD 189733, all of which are late-G or early-K dwarfs with relatively high chromospheric activity indices $\left(\log R_{\mathrm{HK}}^{\prime}>-4.5\right.$; Sing et al. 2016).

\subsection{Additional Impact of Stellar Chromospheres}

In this initial study we examine the effects of heterogeneity purely in a photospheric context. However, we recognize the widespread occurrence of chromospheres in late-type stars, which may be operationally defined as an outer atmospheric region coinciding with the onset of a positive temperature gradient with height (Linsky 1980). In a physical context, chromospheric and coronal regions on the Sun and, by extension, in late-type stars are spatially associated with emergent magnetic flux, i.e., precisely the kind of heterogeneities that affect the interpretation of exoplanet transmission spectra. Chromospheric heating can impact spectral line profile shapes and strengths, including those of key features such as the $\mathrm{Ca}$ II $\mathrm{H}$ and $\mathrm{K}$ resonance lines in the blue-visible and their $\mathrm{UV}$ counterparts, the $\mathrm{Mg}$ II $\mathrm{h}$ and $\mathrm{k}$ resonance lines; the $\mathrm{Ca}$ II infrared triplet lines, the $\mathrm{NaI} \mathrm{D}$ lines and the Balmer lines. Additionally, lower chromospheric and upper photospheric heating can alter the ionization fractions of neutral metal species, notably that of the Fe I lines and $\mathrm{K} \mathrm{I}$ in addition to the concentrations of molecular species such as CO.

Some quantitative insight on the magnitude of the effects of enhanced chromospheric heating on atomic lines is provided by results of long-term studies of solar variability. Livingston et al. (2007) summarizes observations of spectral line variability seen in the Sun-as-a-star in their multi-decadal program from 1974 to 2006. Inspection of the figures in Livingston et al. (2007) reveals, for example, that the peak-to-peak full-disk cycle variations in the $\mathrm{Ca}$ II $\mathrm{K}$ index, defined as the relative strength of the line core in a central $1 \AA$ bandpass, are approximately $25 \%$. The Na I D lines can change by $22 \%$ in central intensity during the solar cycle, while photospheric Fe I lines can exhibit central intensity changes of $\sim 6 \%$. The central depths of both the Ca II IR triplet feature at $8542 \AA$ and $\mathrm{H} \alpha$ also vary in phase with the solar cycle, though at lower relative amplitudes than the $\mathrm{Ca}$ II $\mathrm{K}$ line.

The particular case of the $\mathrm{CO}$ molecule is interesting because its formation and behavior is intimately linked to the inhomogeneous nature of the solar atmosphere. In particular, Ayres (1981) observed that in the presence of localized mechanical heating, $\mathrm{CO}$ molecules begin to disassociate, leading to a decline in radiative cooling. A new equilibrium is only established at higher temperatures where ionized $\mathrm{Ca}$ and $\mathrm{Mg}$ become the dominant radiative coolants in the chromosphere. Outside of these regions, the outer atmospheres exists at a temperature lower than that of the chromospheric temperature minimum with radiative cooling in the $\mathrm{CO}$ bands playing a key role in determining the local thermal structure. As Ayres (1981) concludes, the heterogeneous solar atmosphere is thermally bifurcated between hot chromospheric regions where the CO molecule is depleted and locally cold regions where $\mathrm{CO}$ is enhanced (see also Ayres \& Rabin 1996).

At cooler effective temperatures beginning with the $\mathrm{K}$ dwarfs, $\mathrm{H} \alpha$ becomes a prominent indicator of the presence of chromospheres in emission and absorption (Cram \& Mullan 1979; Cram \& Giampapa 1987). The Ca II core emission and $\mathrm{H} \alpha$ strength are correlated with $\mathrm{K}$ dwarfs that exhibit very weak $\mathrm{H}$ and $\mathrm{K}$ emission and also show weak $\mathrm{H} \alpha$ absorption that is dominated by the photospheric contribution. However, 
among late-K dwarfs and early-M dwarfs, even those objects with weak $\mathrm{Ca}$ II emission still display significant $\mathrm{H} \alpha$ absorption (Robinson et al. 1990). Thus, as Cram \& Giampapa (1987) conclude, the presence of $\mathrm{H} \alpha$ chromospheres in $\mathrm{K}$ and $\mathrm{M}$ dwarfs is ubiquitous - a truly immaculate star in this class may not exist. Therefore, future investigations of specific atomic or molecular features as they may appear in exoplanet transmission spectra may have to include considerations of the impact of chromospheric and coronal heating on their formation, depending on the level of precision required.

\subsection{Promising Paths Forward}

While we find that stellar contamination in transmission spectra of FGK dwarfs is less problematic generally than found for $\mathrm{M}$ dwarfs in Paper I, there are still circumstances when observers should tread carefully. In particular, special care should be taken to distinguish stellar and planetary features in observations involving mid-G to late-K dwarfs-especially active ones-and minute planetary spectral features on the order of tens of ppm or smaller. Here we briefly review approaches that can be useful in these situations.

There are a suite of forward-modeling approaches that provide useful priors for interpreting transmission spectra. In particular, we use variability models in this work to explore spot and facula covering fractions for typically active FGK dwarfs and their associated range of stellar contamination signals. These results can be applied to appropriate FGK host stars, i.e., those with variability amplitudes comparable to the medians tabulated in Table 3. For more or less active stars, the scaling relation coefficients provided in Table 2 can be used to estimate the spot covering fraction, which in turn can be used to approximate the scale of the stellar contamination signal relative to those detailed here. For simplicity, we present observational offsets in Section 4 assuming $D=1 \%$, but these values all scale directly with $D$, so it is trivial to scale them to different transit depths.

The same general forward-modeling approach can be applied to individual interesting stars. Spake et al. (2018), for example, apply the approach detailed here to WASP-107 and find that the scale of the observed helium absorption feature at 10,833 $\AA$ in the transmission spectrum of WASP-107b is much greater that what can be produced by photospheric heterogeneities. These authors also investigate and discount the possibility that the observed helium feature could arise from an inhomogeneous chromosphere, which is an important step for attributing a planetary origin to lines that are also present in chromospheres (see also Cauley et al. 2018).

When this approach is applied to individual host stars, active region crossings observed during exoplanetary transits are particularly helpful. These light-curve anomalies encode the active region size and contrast (i.e., temperature), estimates of which can be obtained with tools like SPOTROD (Béky et al. 2014) or PyTranspot (Juvan et al. 2018). These parameters in turn provide useful inputs to the variability modeling approach that we employ here, refining estimates of the total active region covering fractions corresponding to an observed photometric variability (e.g., Espinoza et al. 2019).

Even more detailed studies of important individual stars can provide further insights. For example, using a combination of high-resolution NIR spectra and long-term photometric monitoring, Gully-Santiago et al. (2017) constrain the spot temperature of the weak-lined T-Tauri star $\mathrm{LkCa} 4$ and trace the temporal evolution of the spot filling factor. By combining both radial velocity and photometric time-series, the StarSim tool (Herrero et al. 2016) can also be used to trace the temporal evolution of photospheric heterogeneities and thus the stellar contamination signals at the time of transit. Studies of the outof-transit stellar spectra flanking transit observations can provide further insights into the relative change in the stellar contamination signal between transits (Zellem et al. 2017).

Finally, transmission spectra retrievals that allow for stellar contamination can be used to distinguish stellar and planetary spectral features. Within a nested sampling framework (Skilling 2006), the Bayesian evidence for models with and without stellar contamination can be straightforwardly compared. Using this approach, Espinoza et al. (2019) concluded that the $\mathrm{TiO}$ absorption features observed in the visual transmission spectrum of WASP-19b are likely produced by unocculted spots in the photosphere of the active G9V host star. Meanwhile, using the same approach, Bixel et al. (2019) found no evidence of stellar contamination in the visual transmission spectrum of WASP-4b, a system similar in most respects but with a less-active host star. In a systematic study of the Sing et al. (2016) sample of hot Jupiters using a joint stellar and planetary retrieval framework, Pinhas et al. (2018) identified a tentative but suggestive trend between the chromospheric activity index $\log R_{\mathrm{HK}}^{\prime}$ and the Bayesian evidence in support of models that allow for stellar contamination features. If confirmed, this finding suggests that $\log R_{\mathrm{HK}}^{\prime}$ can be used to predict whether stellar contamination will affect transmission spectra from a given host star. Along with the trends in stellar contamination features discussed in Section 4.4, systematic trends like these can provide further context for interpreting spectral features in a given transmission spectrum.

\section{Conclusions}

We have presented a study of photospheric heterogeneity in FGK stars and its associated effect on exoplanet transmission spectra in the $0.05-5.5 \mu \mathrm{m}$ wavelength range. The key results of this study are as follows:

1. For both spots and spots+faculae models, rotational variability amplitudes in the Kepler bandpass show a square-root-like dependence on the spot covering fraction, allowing estimates of spot covering fractions to be obtained from observed variabilities.

2. Relative to $\mathbf{M}$ dwarfs, the lower variabilities that are typically observed for FGK stars point to lower active region covering fractions and enable tighter estimates on the covering fractions from rotational variability modeling.

3. We find that the median Kepler variability amplitudes for spectral types $\mathrm{F} 5 \mathrm{~V}-\mathrm{K} 9 \mathrm{~V}$ correspond to spot covering fractions that generally increase with later spectral types, from roughly $0.1 \%$ for $\mathrm{F}$ dwarfs to $2 \%-4 \%$ for late-K dwarfs.

4. If present on the unocculted stellar disk, these heterogeneities primarily impact transmission spectra by increasing transit depths across the studied wavelength range. The largest differences between the stellar contamination spectra that we calculate for spots and spots+faculae models occur at wavelengths $\lesssim 0.5 \mu \mathrm{m}$, for which the spots models predict relatively 
large increases in transit depth, while the spots +faculae models predict strong decreases in transit depth. Thus, transit observations at short wavelengths can be used to constrain the presence of unocculted faculae on the stellar disk.

5. In general, the largest impacts of stellar contamination in transmission spectra are evident at UV and visual wavelengths. We calculate the offsets between blue $(0.4 \mu \mathrm{m})$ and red $(0.9 \mu \mathrm{m})$ visual transit depths owing to stellar contamination. Assuming a nominal transit depth of $1 \%$ and a $30 \mathrm{ppm}$ detection threshold, we find that typically active $\mathrm{G}$ and $\mathrm{K}$ dwarfs can impart detectable visual offsets on transmission spectra.

6. Exploring line offsets in stellar contamination spectra around $\mathrm{H} \alpha$ and the $\mathrm{Na} \mathrm{D}$ and $\mathrm{K}$ doublets, we find that unocculted spots on typically active FGK dwarfs do not alter transit depths detectably, though unocculted faculae in $\mathrm{K}$ dwarfs can decrease transit depths around the $\mathrm{Na} \mathrm{D}$ doublet by a few hundreds of ppm. For more active host stars, we caution that detectable changes may be evident for more atomic features and earlier spectral types, and we suggest that trends in relative strengths of these features can be used to identify their stellar origin.

7. We calculate transit depth offsets at wavelengths of interest for $\mathrm{CH}_{4}, \mathrm{CO}, \mathrm{CO}_{2}, \mathrm{H}_{2} \mathrm{O}, \mathrm{N}_{2} \mathrm{O}, \mathrm{O}_{2}$, and $\mathrm{O}_{3}$ and find that none are detectable for typically active FGK dwarfs, again assuming a $1 \%$ transit depth and $30 \mathrm{ppm}$ detection threshold. Of these, the largest offsets are apparent at wavelengths of interest for $\mathrm{O}_{2}, \mathrm{H}_{2} \mathrm{O}$, and $\mathrm{CH}_{4}$, which have molecular bands at shorter wavelengths. Larger offsets are possible for more active host stars, and so we suggest that future works exercise care when studying these features in the atmospheres of exoplanets hosted by active $\mathrm{G}$ and $\mathrm{K}$ stars.

8. Defining the deviation of the visual stellar contamination spectrum from a simple slope as a proxy for $\mathrm{TiO} / \mathrm{VO}$ features, we find that stellar $\mathrm{TiO} / \mathrm{VO}$ features in transmission spectra are potentially detectable for typically active late-K dwarfs and, for active stars, can be apparent for spectral types as early as G8V.

9. Taking the long view, we explore stellar contamination in an Earth-Sun analog system and find that transit depth offsets due to stellar contamination at wavelengths of interest for important atmospheric molecular absorbers are $\lesssim 20 \mathrm{ppb}$, roughly an order of magnitude lower than the scale of a planetary atmospheric feature covering a single scale height.

The whole of this analysis shows that stellar contamination in transmission spectra of FGK-hosted exoplanets is generally less problematic than for exoplanets orbiting $\mathbf{M}$ dwarfs. The impact of the TLS effect is most prominent at shorter wavelengths. While it can produce detectable slopes in visual transmission spectra from $\mathrm{G}$ and $\mathrm{K}$ dwarfs and, for more-active late- $\mathrm{G}$ and $\mathrm{K}$ dwarfs, detectable offsets at wavelengths of interest for $\mathrm{TiO} / \mathrm{VO}$, TLS signals are generally minor at wavelengths of planetary atomic and molecular features. This bodes well for high-precision observations of these targets, including those expected to be discovered by the recently launched TESS mission (Ricker et al. 2015), with current ground- and space-based facilities and near-future facilities like the JWST. However, within the parameter space that we explore, more care should be exercised for observations at shorter wavelengths and those with host stars that are more active or of later spectral types.

B.R. acknowledges support from the National Science Foundation Graduate Research Fellowship Program under grant No. DGE-1143953. D.A. acknowledges support from the Max Planck Institute for Astronomy, Heidelberg, for a sabbatical visit. M.S.G. thanks the Lunar and Planetary Lab at the University of Arizona for hosting him during his sabbatical leave. We thank the anonymous referee for their constructive comments. The results reported herein benefited from collaborations and/or information exchange within NASA's Nexus for Exoplanet System Science (NExSS) research coordination network sponsored by NASA's Science Mission Directorate. The National Solar Observatory is operated by AURA under a cooperative agreement with the National Science Foundation. This research has made use of NASA's Astrophysics Data System.

Software: Astropy (Astropy Collaboration et al. 2013, 2018), Matplotlib (Hunter 2007), NumPy (van der Walt et al. 2011), SciPy (Jones et al. 2001).

\section{ORCID iDs}

Benjamin V. Rackham (iD https://orcid.org/0000-00023627-1676

Dániel Apai (ib https://orcid.org/0000-0003-3714-5855

Mark S. Giampapa (iD https://orcid.org/0000-0002-2132-5264

\section{References}

Albrecht, S., Winn, J. N., Johnson, J. A., et al. 2012, ApJ, 757, 18

Andretta, V., Giampapa, M. S., Covino, E., Reiners, A., \& Beeck, B. 2017, ApJ, 839, 97

Apai, D., Rackham, B. V., Giampapa, M. S., et al. 2018, arXiv:1803.08708

Astropy Collaboration, Robitaille, T. P., Tollerud, E. J., et al. 2013, A\&A, 558, A33

Astropy Collaboratio, Price-Whelan, A. M., Sipőcz, B. M., et al. 2018, AJ, 156,123

Ayres, T. R. 1981, ApJ, 244, 1064

Ayres, T. R., \& Rabin, D. 1996, ApJ, 460, 1042

Babcock, H. W. 1961, ApJ, 133, 572

Baliunas, S. L., Donahue, R. A., Soon, W. H., et al. 1995, ApJ, 438, 269

Barstow, J. K., Aigrain, S., Irwin, P. G. J., \& Sing, D. K. 2017, ApJ, 834, 50

Basri, G., Walkowicz, L. M., Batalha, N., et al. 2010, ApJL, 713, L155

Basri, G., Walkowicz, L. M., Batalha, N., et al. 2011, AJ, 141, 20

Basri, G., Walkowicz, L. M., \& Reiners, A. 2013, ApJ, 769, 37

Bean, J. L., Miller-Ricci Kempton, E., \& Homeier, D. 2010, Natur, 468, 669

Béky, B., Kipping, D. M., \& Holman, M. J. 2014, MNRAS, 442, 3686

Ben-Ami, S., López-Morales, M., Garcia-Mejia, J., Gonzalez Abad, G., \& Szentgyorgyi, A. 2018, ApJ, 861, 79

Berdyugina, S. V. 2005, LRSP, 2, 8

Berta, Z. K., Charbonneau, D., Bean, J., et al. 2011, ApJ, 736, 12

Birch, K. P., \& Downs, M. J. 1994, Metro, 31, 315

Bixel, A., Rackham, B. V., Apai, D., et al. 2019, AJ, 157, 68

Brogi, M., Snellen, I. A. G., de Kok, R. J., et al. 2012, Natur, 486, 502

Brown, T. M. 2001, ApJ, 553, 1006

Carter, J. A., Winn, J. N., Holman, M. J., et al. 2011, ApJ, 730, 82

Cauley, P. W., Kuckein, C., Redfield, S., et al. 2018, AJ, 156, 189

Cauley, P. W., Redfield, S., \& Jensen, A. G. 2017, AJ, 153, 217

Charbonneau, D., Brown, T. M., Noyes, R. W., \& Gilliland, R. L. 2002, ApJ, 568,377

Claret, A. 2000, A\&A, 363, 1081

Collier Cameron, A. 2017, in Exoplanets, ed. H. Deeg \& J. Belmonte (Cham: Springer), 23

Cram, L. E., \& Giampapa, M. S. 1987, ApJ, 323, 316

Cram, L. E., \& Mullan, D. J. 1979, ApJ, 234, 579

Dawson, R. I. 2014, ApJL, 790, L31

Deupree, R. G. 2011, ApJ, 735, 69

Dravins, D., Gustavsson, M., \& Ludwig, H.-G. 2018, A\&A, 616, A144 
Dravins, D., Ludwig, H.-G., Dahlén, E., \& Pazira, H. 2017a, A\&A, 605, A90 Dravins, D., Ludwig, H.-G., Dahlén, E., \& Pazira, H. 2017b, A\&A, 605, A91 Ehrenreich, D., Tinetti, G., Lecavelier Des Etangs, A., Vidal-Madjar, A., \& Selsis, F. 2006, A\&A, 448, 379

Espinoza, N., Rackham, B. V., Jordán, A., et al. 2019, MNRAS, 482, 2065 Evans, T. M., Sing, D. K., Kataria, T., et al. 2017, Natur, 548, 58

Evans, T. M., Sing, D. K., Wakeford, H. R., et al. 2016, ApJL, 822, L4 Fortney, J. J., Shabram, M., Showman, A. P., et al. 2010, ApJ, 709, 1396 Fraine, J., Deming, D., Benneke, B., et al. 2014, Natur, 513, 526 Giampapa, M. S., \& Rosner, R. 1984, ApJL, 286, L19 Gibson, N. P., Aigrain, S., Barstow, J. K., et al. 2013, MNRAS, 436, 2974 Gondoin, P. 2008, A\&A, 478, 883

Greene, T. P., Line, M. R., Montero, C., et al. 2016, ApJ, 817, 17

Gully-Santiago, M. A., Herczeg, G. J., Czekala, I., et al. 2017, ApJ, 836, 200 Hathaway, D. H. 2011, SoPh, 273, 221

Herrero, E., Ribas, I., Jordi, C., et al. 2016, A\&A, 586, A131

Hill, C., Yurchenko, S. N., \& Tennyson, J. 2013, Icar, 226, 1673

Hirano, T., Narita, N., Shporer, A., et al. 2011, PASJ, 63, 531

Hubbard, W. B., Fortney, J. J., Lunine, J. I., et al. 2001, ApJ, 560, 413

Huitson, C. M., Désert, J.-M., Bean, J. L., et al. 2017, AJ, 154, 95

Huitson, C. M., Sing, D. K., Pont, F., et al. 2013, MNRAS, 434, 3252

Hunter, J. D. 2007, CSE, 9, 90

Husser, T.-O., Wende-von Berg, S., Dreizler, S., et al. 2013, A\&A, 553, A6

Jackson, R. J., \& Jeffries, R. D. 2012, MNRAS, 423, 2966

Jackson, R. J., \& Jeffries, R. D. 2013, MNRAS, 431, 1883

Jensen, A. G., Redfield, S., Endl, M., et al. 2012, ApJ, 751, 86

Jones, E., Oliphant, T., \& Peterson, P. 2001, SciPy:Open source scientific tools for Python, http://www.scipy.org/

Jordán, A., Espinoza, N., Rabus, M., et al. 2013, ApJ, 778, 184

Juvan, I. G., Lendl, M., Cubillos, P. E., et al. 2018, A\&A, 610, A15

Kaltenegger, L., \& Traub, W. A. 2009, ApJ, 698, 519

Keller, C. U., Schüssler, M., Vögler, A., \& Zakharov, V. 2004, ApJL, 607, L59

Kobel, P., Solanki, S. K., \& Borrero, J. M. 2011, A\&A, 531, A112

Kraft, R. P. 1967, ApJ, 150, 551

Kreidberg, L., Bean, J. L., Désert, J.-M., et al. 2014a, ApJL, 793, L27

Kreidberg, L., Bean, J. L., Désert, J.-M., et al. 2014b, Natur, 505, 69

Kreidberg, L., Line, M. R., Bean, J. L., et al. 2015, ApJ, 814, 66

Lecavelier Des Etangs, A., Pont, F., Vidal-Madjar, A., \& Sing, D. 2008, A\&A, 481, L83

Linsky, J. L. 1980, ARA\&A, 18, 439

Lites, B. W., Scharmer, G. B., Berger, T. E., \& Title, A. M. 2004, SoPh, 221,65

Livingston, W., Wallace, L., White, O. R., \& Giampapa, M. S. 2007, ApJ, 657, 1137

Llama, J., \& Shkolnik, E. L. 2015, ApJ, 802, 41

Makarov, V. I., \& Makarova, V. V. 1996, SoPh, 163, 267

Mallonn, M., Herrero, E., Juvan, I. G., et al. 2018, A\&A, 614, A35

Mancini, L., Ciceri, S., Chen, G., et al. 2013, MNRAS, 436, 2

Mandal, S., Hegde, M., Samanta, T., et al. 2017, A\&A, 601, A106

Maunder, E. W. 1904, MNRAS, 64, 747

Maunder, E. W. 1922, MNRAS, 82, 534

Mazeh, T., Perets, H. B., McQuillan, A., \& Goldstein, E. S. 2015, ApJ, 801, 3

McCullough, P. R., Crouzet, N., Deming, D., \& Madhusudhan, N. 2014, ApJ, 791,55

McQuillan, A., Aigrain, S., \& Mazeh, T. 2013, MNRAS, 432, 1203

McQuillan, A., Mazeh, T., \& Aigrain, S. 2014, ApJS, 211, 24

Miller-Ricci, E., Seager, S., \& Sasselov, D. 2009, ApJ, 690, 1056

Morris, B. M., Hebb, L., Davenport, J. R. A., Rohn, G., \& Hawley, S. L. 2017, ApJ, 846, 99

Narita, N., Fukui, A., Ikoma, M., et al. 2013, ApJ, 773, 144

Neff, J. E., O’Neal, D., \& Saar, S. H. 1995, ApJ, 452, 879

Nikolov, N., Sing, D. K., Fortney, J. J., et al. 2018, Natur, 557, 526

Norris, C. M., Beeck, B., Unruh, Y. C., et al. 2017, A\&A, 605, A45

Noyes, R. W., Hartmann, L. W., Baliunas, S. L., Duncan, D. K., \& Vaughan, A. H. 1984, ApJ, 279, 763

Oshagh, M., Santos, N. C., Ehrenreich, D., et al. 2014, A\&A, 568, A99

Pallé, E., Zapatero Osorio, M. R., Barrena, R., Montañés-Rodríguez, P., \& Martín, E. L. 2009, Natur, 459, 814
Parker, E. N. 1955, ApJ, 121, 491

Parviainen, H., Pallé, E., Chen, G., et al. 2018, A\&A, 609, A33

Pecaut, M. J., \& Mamajek, E. E. 2013, ApJS, 208, 9

Pinhas, A., Madhusudhan, N., Gandhi, S., \& MacDonald, R. 2019, MNRAS, 482, 1485

Pinhas, A., Rackham, B. V., Madhusudhan, N., \& Apai, D. 2018, MNRAS, 480, 5314

Pizzolato, N., Maggio, A., Micela, G., Sciortino, S., \& Ventura, P. 2003, A\&A, 397, 147

Pont, F., Knutson, H., Gilliland, R. L., Moutou, C., \& Charbonneau, D. 2008 MNRAS, 385, 109

Pont, F., Sing, D. K., Gibson, N. P., et al. 2013, MNRAS, 432, 2917

Rackham, B., Espinoza, N., Apai, D., et al. 2017, ApJ, 834, 151

Rackham, B. V., Apai, D., \& Giampapa, M. S. 2018, ApJ, 853, 122

Radick, R. R., Hartmann, L., Mihalas, D., et al. 1982, PASP, 94, 934

Ramsey, L. W., \& Nations, H. L. 1980, ApJL, 239, L121

Ricker, G. R., Winn, J. N., Vanderspek, R., et al. 2015, JATIS, 1, 014003

Robinson, R. D., Cram, L. E., \& Giampapa, M. S. 1990, ApJS, 74, 891

Rodler, F., \& López-Morales, M. 2014, ApJ, 781, 54

Rodler, F., Lopez-Morales, M., \& Ribas, I. 2012, ApJL, 753, L25

Ruzmaikin, A. 2001, SSRv, 95, 43

Sanchis-Ojeda, R., \& Winn, J. N. 2011, ApJ, 743, 61

Scandariato, G., Nascimbeni, V., Lanza, A. F., et al. 2017, A\&A, 606, A134

Schlaufman, K. C. 2010, ApJ, 719, 602

Schmitt, J. H. M. M. 2001, in ASP Conf. Ser. 248, Magnetic Fields Across the Hertzsprung-Russell Diagram, ed. G. Mathys, S. K. Solanki, \& D. T. Wickramasinghe (San Francisco, CA: ASP), 199

Schmitt, J. H. M. M., \& Rosner, R. 1983, ApJ, 265, 901

Schröder, C., Reiners, A., \& Schmitt, J. H. M. M. 2009, A\&A, 493, 1099

Seager, S., \& Sasselov, D. D. 2000, ApJ, 537, 916

Sedaghati, E., Boffin, H. M. J., MacDonald, R. J., et al. 2017, Natur, 549, 238

Shapiro, A. I., Solanki, S. K., Krivova, N. A., et al. 2014, A\&A, 569, A38

Shapiro, A. I., Solanki, S. K., Krivova, N. A., Yeo, K. L., \& Schmutz, W. K. 2016, A\&A, 589, A46

Siess, L., Dufour, E., \& Forestini, M. 2000, A\&A, 358, 593

Sing, D. K., Fortney, J. J., Nikolov, N., et al. 2016, Natur, 529, 59

Sing, D. K., Huitson, C. M., Lopez-Morales, M., et al. 2012, MNRAS, 426, 1663

Sing, D. K., Pont, F., Aigrain, S., et al. 2011, MNRAS, 416, 1443

Skilling, J. 2006, BayAn, 1, 833

Snellen, I. A. G., de Kok, R. J., de Mooij, E. J. W., \& Albrecht, S. 2010, Natur, 465,1049

Snellen, I. A. G., de Kok, R. J., le Poole, R., Brogi, M., \& Birkby, J. 2013, ApJ, 764,182

Solanki, S. K. 2003, A\&ARv, 11, 153

Spake, J. J., Sing, D. K., Evans, T. M., et al. 2018, Natur, 557, 68

Spruit, H. C. 1976, SoPh, 50, 269

Stevenson, K. B., Bean, J. L., Seifahrt, A., et al. 2016, ApJ, 817, 141

Strassmeier, K. G. 2009, A\&ARv, 17, 251

Tregloan-Reed, J., Southworth, J., \& Tappert, C. 2013, MNRAS, 428, 3671

van der Walt, S., Colbert, S. C., \& Varoquaux, G. 2011, CSE, 13, 22

Vogt, S. S. 1979, PASP, 91, 616

Vogt, S. S. 1981, ApJ, 250, 327

Wakeford, H. R., Sing, D. K., Deming, D., et al. 2018, AJ, 155, 29

Wakeford, H. R., Sing, D. K., Kataria, T., et al. 2017, Sci, 356, 628

Walkowicz, L. M., \& Basri, G. S. 2013, MNRAS, 436, 1883

Wallace, L., Bernath, P., Livingston, W., et al. 1995, Sci, 268, 1155

Willson, R. C., Hudson, H. S., Frohlich, C., \& Brusa, R. W. 1986, Sci, 234, 1114

Winn, J. N., Fabrycky, D., Albrecht, S., \& Johnson, J. A. 2010a, ApJL, 718, L145

Winn, J. N., Johnson, J. A., Howard, A. W., et al. 2010b, ApJL, 723, L223

Winn, J. N., Petigura, E. A., Morton, T. D., et al. 2017, AJ, 154, 270

Yee, S. W., Petigura, E. A., Fulton, B. J., et al. 2018, AJ, 155, 255

Zellem, R. T., Swain, M. R., Roudier, G., et al. 2017, ApJ, 844, 27

Zhang, Z., Zhou, Y., Rackham, B. V., \& Apai, D. 2018, AJ, 156, 178 